TRANSACTIONS OF THE

AMERICAN MATHEMATICAL SOCIETY

Volume 350, Number 2, February 1998, Pages 539-558

S 0002-9947(98)01865-0

\title{
EXTREMAL VECTORS AND INVARIANT SUBSPACES
}

\author{
SHAMIM ANSARI AND PER ENFLO
}

\begin{abstract}
For a bounded linear operator on Hilbert space we define a sequence of so-called minimal vectors in connection with invariant subspaces and show that this presents a new approach to invariant subspaces. In particular, we show that for any compact operator $K$ some weak limit of the sequence of minimal vectors is noncyclic for all operators commuting with $K$ and that for any normal operator $N$, the norm limit of the sequence of minimal vectors is noncyclic for all operators commuting with $N$. Thus, we give a new and more constructive proof of existence of invariant subspaces.

The sequence of minimal vectors does not seem to converge in norm for an arbitrary bounded linear operator. We will prove that if $T$ belongs to a certain class $\mathcal{C}$ of operators, then the sequence of such vectors converges in norm, and that if $T$ belongs to a subclass of $\mathcal{C}$, then the norm limit is cyclic.
\end{abstract}

\section{INTRODUCTION}

In this paper we will study different types of extremal vectors for operators on Hilbert space and their connection to invariant subspaces. We present a new method to find invariant subspaces, which we feel is more constructive then the known ones and which gives hyperinvariant subspaces for all compact and all normal operators in a unified way. We feel that the method may give invariant subspaces for several other classes of operators, but so far we have only succeeded in proving this under extra assumptions.

The second author started this study by considering the best approximate solutions of the equations $T^{n} y=x_{0}$ for the case when $T$ has dense range and $x_{0}$ is not in the range of $T$. More precisely, he considered the $y_{n}$ of smallest norm such that $\left\|T^{n} y_{n}-x_{0}\right\| \leq \epsilon$. Using these backward minimal points, $T^{n} y_{n}$ 's, he proved Theorem 4. The first author introduced forward minimal points $v_{n}$ 's such that $\left\|v_{n}-x_{0}\right\| \leq \epsilon$ and $\left\|T^{n} v_{n}\right\|$ is minimal. This gives even a simpler proof of Theorem 4 , and there is a duality between the forward and the backward minimal points as given in Proposition 2. In this paper we also study geometric properties of $v_{n}$ and $T^{n} y_{n}$ involving scalar products and limit points. These geometric properties have a connection to invariant subspaces. We show that $\left(T^{n} y_{n}\right)$ converges in norm for normal operators. In relation to the norm convergence of the extremal vectors, we introduce a new class of operators, called operators of class $\mathcal{R}$. This class generalizes $C_{1}$-contractions, and we feel that it is of interest in its own right too. Sections 1-2 are due to the second author, $3-4$ are due to the first and the appendix is due to both.

Received by the editors October 16, 1995.

1991 Mathematics Subject Classification. Primary 47A15.

Partially supported by NSF grant number 441003. 


\section{Extremal VECTORS AND THEIR PROPERTIES}

In this paper $H$ will denote a Hilbert space over the field of complex numbers, $X$ a reflexive strictly convex Banach space over the field of complex numbers. For any operator $T: X \rightarrow X, r=r(T)$ will represent the spectral radius of $T$ and $\mathcal{R}(T)$ the range of $T$.

Backward Minimal Vectors. Let $T: H \rightarrow H$ be a bounded operator with dense range. Let $x_{0} \in H$ and $\epsilon>0$ with $\epsilon<\left\|x_{0}\right\|$. There is a unique vector $y_{n, x_{0}}^{\epsilon}$ such that $\left\|T^{n} y_{n, x_{0}}^{\epsilon}-x_{0}\right\| \leq \epsilon$ and

$$
\left\|y_{n, x_{0}}^{\epsilon}\right\|=\inf \left\{\|y\|:\left\|T^{n} y-x_{0}\right\| \leq \epsilon\right\} .
$$

The points $y_{n, x_{0}}^{\epsilon}$ are called backward minimal points.

Forward Minimal Vectors. Let $T: H \rightarrow H$ be an injective bounded operator. Let $x_{0} \in H$ and $\epsilon>0$ with $\epsilon<\left\|x_{0}\right\|$. There is a unique vector $v_{n, x_{0}}^{\epsilon}$ such that $\left\|v_{n, x_{0}}^{\epsilon}-x_{0}\right\| \leq \epsilon$ and

$$
\left\|T^{n} v_{n, x_{0}}^{\epsilon}\right\|=\inf \left\{\left\|T^{n} v\right\|:\left\|v-x_{0}\right\| \leq \epsilon\right\} .
$$

The points $T^{n} v_{n, x_{0}}^{\epsilon}$ are called forward minimal points.

When there is no ambiguity, we will drop some of the superscripts and subscripts from $y_{n, x_{0}}^{\epsilon}$ and $v_{n, x_{0}}^{\epsilon}$. Some times the vectors $T^{n} y_{n}$ and $v_{n}$ will also be referred as backward, respectively, forward minimal vectors. It is clear that $\left\|T^{n} y_{n, x_{0}}^{\epsilon}-x_{0}\right\|=\epsilon$ and $\left\|v_{n}-x_{0}\right\|=\epsilon$.

Theorem 1 (Orthogonality Equations). There exist constants $\delta_{\epsilon}<0$ and $\delta_{\epsilon}^{\prime}<$ 0 such that

$$
\begin{aligned}
& y_{\epsilon}=\delta_{\epsilon} T^{*}\left(T y_{\epsilon}-x_{0}\right), \\
& \left(v_{\epsilon}-x_{0}\right)=\delta_{\epsilon}^{\prime} T^{*} T v_{\epsilon} .
\end{aligned}
$$

Proof. We will prove only the first equation, since the proof of the second equation is similar. Note that for any vectors $u, w$, if $\operatorname{Re}(u, w)<0$, then the function $r(t)=\|u+t w\|$ is decreasing over $\left[0, t_{0}\right]$ for some $t_{0}>0$, whereas if $\operatorname{Re}(u, w) \geq 0$, then the function $r(t)=\|u+t z\|$ is increasing over $[0, \infty)$. Thus, if we have $\operatorname{Re}\left(T^{*}\left(T y_{\epsilon}-x_{0}\right), z\right)<0$, then $r(t)=\left\|\left(T y_{\epsilon}-x_{0}\right)+t T z\right\|$ is decreasing over $\left[0, t_{0}\right]$ for some $t_{0}$. So, $\left\|T y_{\epsilon}-x_{0}\right\| \geq\left\|\left(T y_{\epsilon}-x_{0}\right)+t T z\right\|$ for all $t \in\left[0, t_{0}\right]$. By the definition of $y_{\epsilon}$ it follows that $\left\|y_{\epsilon}+t z\right\| \geq\left\|y_{\epsilon}\right\|$ for $t \in\left[0, t_{0}\right]$. Hence, $\operatorname{Re}\left(y_{\epsilon}, z\right) \geq 0$. Since $z$ is arbitrary it follows that for some $\delta_{\epsilon}<0$ we have $y_{\epsilon}=\delta_{\epsilon} \cdot T^{*}\left(T y_{\epsilon}-x_{0}\right)$.

Remark 1. From the orthogonality equations we obtain

$$
\begin{aligned}
T^{n} y_{n} & =-\delta_{n}\left(I-\delta_{n} T^{n} T^{* n}\right)^{-1} T^{n} T^{* n} x_{0}, \\
v_{n} & =\left(I-\delta_{n} T^{* n} T^{n}\right)^{-1} x_{0} .
\end{aligned}
$$

Let $A_{n}=-\delta_{n}\left(I-\delta_{n} T^{n} T^{* n}\right)^{-1} T^{n} T^{* n}$ and $B_{n}=\left(I-\delta_{n} T^{* n} T^{n}\right)^{-1}$. Then $A_{n}$ and $B_{n}$ are positive operators. $\left\|A_{n}\right\|<1$ and $\left\|B_{n}\right\|=1$ for all $n$. Let $f_{n}(z)=\frac{-\delta_{n} \cdot z}{1-\delta_{n} \cdot z}$. Then $f_{n}(z)$ is analytic in a neighborhood of $\sigma\left(T^{n} T^{* n}\right)$ and $\sigma\left(A_{n}\right)=f_{n}\left(\sigma\left(T^{n} T^{* n}\right)\right)$. The maximum point of $f\left(\sigma\left(T^{n} T^{* n}\right)\right)$ is $\frac{-\delta_{n} \cdot\left\|T^{n}\right\|^{2}}{1-\delta_{n} \cdot\left\|T^{n}\right\|^{2}}$. It follows that $\left\|A_{n}\right\|=\frac{-\delta_{n} \cdot\left\|T^{n}\right\|^{2}}{1-\delta_{n} \cdot\left\|T^{n}\right\|^{2}}<$ 1. Now let $g_{n}(z)=\frac{1}{1-\delta_{n} \cdot z}$. Then, $g_{n}$ is analytic in a neighborhood of $\sigma\left(T^{n} T^{* n}\right)$, and $\sigma\left(B_{n}\right)=g_{n}\left(\sigma\left(T^{n} T^{* n}\right)\right.$. Since $0 \in \sigma\left(T^{n} T^{* n}\right)$, it can be verified that the maximum point of $g_{n}\left(\sigma\left(T^{n} T^{* n}\right)\right)$ is 1 . Hence, $\left\|B_{n}\right\|=1$. 
Theorem 2. Let $x_{0} \in H$ and $x_{0} \neq 0$. The functions $\epsilon \longmapsto y_{\epsilon}$ and $\epsilon \longmapsto T v$ are analytic on $\left(0,\left\|x_{0}\right\|\right)$.

Proof. From the orthogonality equation for $y_{\epsilon}$ we have $y_{\epsilon}=-\delta_{\epsilon}\left(I-\delta_{\epsilon} T^{*} T\right)^{-1} T^{*} x_{0}$. So, clearly the analyticity of the function $\epsilon \longmapsto \delta_{\epsilon}$ implies the analyticity of $\epsilon \longmapsto y_{\epsilon}$. To show that $\epsilon \longmapsto \delta_{\epsilon}$ is analytic we will prove that its inverse function $\delta_{\epsilon} \longmapsto \epsilon$ is analytic. (Note that this inverse function is well defined by the uniqueness of $y_{\epsilon}$.) We have

$$
\begin{gathered}
\epsilon=\left\|T y_{\epsilon}-x_{0}\right\|=\left\|-\delta_{\epsilon} T\left(I-\delta_{\epsilon} T^{*} T\right)^{-1} T^{*} x_{0}-x_{0}\right\|, \\
\epsilon^{2}=\left\|-\delta_{\epsilon} T\left(I-\delta_{\epsilon} T^{*} T\right)^{-1} T^{*} x_{0}-x_{0}\right\|^{2} .
\end{gathered}
$$

It follows that $\delta_{\epsilon} \longmapsto \epsilon^{2}$ is analytic and injective. Hence, $\delta_{\epsilon} \longmapsto \epsilon$ is analytic.

Proposition 1. If $\left\|T y-x_{0}\right\| \leq \epsilon$, then $\left|\left\langle y, y_{\epsilon}\right\rangle\right| \geq\left\|y_{\epsilon}\right\|^{2}$.

Proof. Consider the plane spanned by $x_{0}$ and $T y_{\epsilon}$. Assume $z=a y_{\epsilon}+r$, where $a<1$ and $r \perp y_{\epsilon}$. We get $T z=a T y_{\epsilon}+T r$. Put $T r=u+v$, where $u \in \operatorname{span}\left\{x_{0}, T y_{\epsilon}\right\}$ and $v \perp \operatorname{span}\left\{x_{0}, T y_{\epsilon}\right\}$. We know that $u \perp x_{0}-T y_{\epsilon}$. We get $\left\|x_{0}-T z\right\| \geq\left\|x_{0}-a T y_{\epsilon}-u\right\|$. We have $\left\langle x_{0}-T y_{\epsilon}, T y_{\epsilon}\right\rangle>0$. Thus, $\left\|x_{0}-T y_{\epsilon}\right\|^{2}=\left\langle x_{0}-T y_{\epsilon}, x_{0}-T y_{\epsilon}\right\rangle<$ $\left\langle x_{0}-T y_{\epsilon}, x_{)}-a T y_{\epsilon}\right\rangle$. Since $\left\langle x_{0}-a T y_{\epsilon}, x_{0}-T y_{\epsilon}\right\rangle>\epsilon$ and $u \perp x_{0}-T y_{\epsilon}$ we get $\left\|x_{0}-T z\right\| \geq \epsilon$.

Theorem 3. A. If $T$ has a dense range, then $\left\langle T y_{\epsilon}-x_{0}, T y_{\epsilon}\right\rangle \neq 0$.

B. For any bounded operator $T$ with dense range, if $x_{0} \notin \mathcal{R}(T)$, then

$$
\left\langle\frac{T y_{\epsilon_{n}}-x_{0}}{\left\|T y_{\epsilon_{n}}-x_{0}\right\|}, \frac{T y_{\epsilon_{n}}}{\left\|T y_{\epsilon_{n}}\right\|}\right\rangle \rightarrow 0
$$

for some sequence $\left(\epsilon_{n}\right)$ converging to 0 .

Proof. A. $\left\langle T y_{\epsilon}-x_{0}, T y_{\epsilon}\right\rangle=\left\langle T^{*}\left(T y_{\epsilon}-x_{0}\right), y_{\epsilon}\right\rangle=\left\langle\delta_{\epsilon}^{-1} y_{\epsilon}, y_{\epsilon}\right\rangle \neq 0$.

B. Let $\epsilon>0$. Let $\theta_{\epsilon}$ be the angle between the vectors $T y_{\epsilon}-x_{0}$ and $T y_{\epsilon}$. Then there exists $t>0$ such that $\left\|x_{0}-(1+t) T y_{\epsilon}\right\|=\epsilon \cdot \sin \theta_{\epsilon}$. Let us see that $t<s \cdot \epsilon$ for some constant $s>0$. We have $(1+t)\left\|T y_{\epsilon}\right\| \leq\left\|T y_{\epsilon}\right\|+2 \epsilon$. So, $t\left\|T y_{\epsilon}\right\| \leq 2 \epsilon$ and $t \leq \frac{2 \epsilon}{\left\|x_{0}\right\|-\epsilon}<s \cdot \epsilon$ for some $s>0$.

Now let $\epsilon_{1}^{\prime}=\epsilon \cdot \sin \theta_{\epsilon}$. Then, $\left\|y_{\epsilon_{1}^{\prime}}\right\| \leq(1+t)\left\|y_{\epsilon}\right\|<(1+s \epsilon)\left\|y_{\epsilon}\right\|$. For $n \geq 2$ let $\epsilon_{n}^{\prime}=\epsilon_{n-1}^{\prime} \cdot \sin \theta_{n-1}$. Applying the above argument repeatedly, we get $\left\|y_{\epsilon_{n}^{\prime}}\right\| \leq$ $\left(1+s \epsilon_{0}\right)\left(1+\epsilon_{1}^{\prime}\right) \cdots\left(1+3 \epsilon_{n}^{\prime}\right)\left\|y_{\epsilon_{0}}\right\|$. We will prove that $\theta_{n_{k}} \rightarrow \frac{\pi}{2}$ for some subsequence $\left(n_{k}\right)$ of natural numbers. If not, then $\theta_{n}>\frac{\pi}{2}+\delta$ for some $\delta>0$ and for all $n \geq n_{0}$ for some $n_{0}$. So, there exists $r$ such that $0<\sin \theta_{n}<r<1$ for all $n \geq n_{0}$. Then $\left(1+s \epsilon_{0}\right)\left(1+s \epsilon_{1}^{\prime}\right) \ldots\left(1+s \epsilon_{n-1}^{\prime}\right) \leq M \cdot \prod_{m=n_{0}}^{\infty}\left(1+s \epsilon_{0} r^{m}\right)=K$ for some constants $M$ and $K$. So, $\left\|y_{\epsilon_{n}^{\prime}}\right\|<K\left\|y_{\epsilon_{0}}\right\|$ for all $n$. Let $\left(y_{n_{k}}\right)$ be a weakly convergent subsequence, converging weakly to $y_{0}$. Then, $T y_{n_{k}} \rightarrow T y_{0}$ and $\left\|T y_{n_{k}}-x_{0}\right\|=\epsilon_{n_{k}} \rightarrow 0$. So, $\left\|T y_{0}-x_{0}\right\|=0$. That is, $T y_{0}=x_{0}$. This contradiction proves that $\theta_{n_{k}} \rightarrow \frac{\pi}{2}$ for some subsequence $n_{k}$. Let $e_{k}=\epsilon_{n_{k}}$. We now claim that $e_{k} \rightarrow 0$. If not, then $\epsilon_{k} \rightarrow \epsilon_{0}$ for some $\epsilon_{0}>0$. By Theorem 2, $y_{\epsilon_{k}} \rightarrow y_{\epsilon_{0}}$. So, $T y_{\epsilon_{k}} \rightarrow T y_{\epsilon_{0}}$. It follows that $\left\langle T y_{\epsilon_{0}}-x_{0}, T y_{\epsilon_{0}}\right\rangle=0$, which contradicts part A. 


\section{INVARIANT SUBSPACES}

Lemma 1. If $T$ is quasinilpotent, then $\frac{\left\|y_{n_{k}-1}\right\|}{\left\|y_{n_{k}}\right\|} \rightarrow 0$ as $k \rightarrow 0$ for some subsequence $\left(n_{k}\right)$.

Proof. Suppose not. Then there is a $t>0$ such that $\frac{\left\|y_{n-1}\right\|}{\left\|y_{n}\right\|}>t$ for all $n>0$. So, $\left\|y_{1}\right\| \geq t\left\|y_{2}\right\| \geq \ldots \geq t^{n-1}\left\|y_{n}\right\|$ for all $n>0$. By the minimality of $\left\|y_{1}\right\|$, $\left\|T^{n-1} y_{n}\right\| \geq\left\|y_{1}\right\|$. So we have $\left\|T^{n-1}\right\|\left\|y_{n}\right\| \geq\left\|T^{n-1} y_{n}\right\| \geq\left\|y_{1}\right\| \geq t^{n-1}\left\|y_{n}\right\|$. Therefore, $\left\|T^{n-1}\right\| \geq t^{n-1}$ for all $n>0$. This contradicts that $\sigma(T)=\{0\}$.

Theorem 4. Every compact operator has a hyperinvariant subspace.

Proof. Let $K$ be a compact operator. If $\overline{\mathcal{R}(K)} \neq H$, then $\overline{\mathcal{R}(K)}$ is a hyperinvariant subspace for $K$. So, we will assume that $\mathcal{R}(K)$ is dense. Also if $K$ has nonzero eigenvalues, then the eigenspace corresponding to each eigenvalue is a hyperinvariant subspace for $K$. So, we can assume that $K$ has no eigenvalues, i.e. $\sigma(K)=\{0\}$.

Let $x_{0} \in H$ with $\left\|x_{0}\right\| \neq 0$ and $0<\epsilon<\left\|x_{0}\right\|$. Let $y_{n}$ be the backward minimal points with respect to $\left(x_{0}, \epsilon, K^{n}\right)$. Let $T$ be any operator commuting with $K$, with $\|T\|=1$. Let $\frac{\left\|y_{n_{k}-1}\right\|}{\left\|y_{n_{k}}\right\|} \rightarrow 0$. We may assume, by passing to a subsequence if necessary, that $K^{n_{k}} y_{n_{k}} \rightarrow y_{0}$ weakly and $K^{n_{k}-1} y_{n_{k}-1} \rightarrow z$ weakly. Then $T K^{n_{k}} y_{n_{k}-1} \rightarrow T K z$ in norm. Let $\alpha_{n_{k}}$ be scalars and $\gamma_{n_{k}}$ be vectors with $\gamma_{n_{k}} \perp y_{n_{k}}$ such that

$$
\begin{aligned}
T y_{n_{k}-1} & =\alpha_{n_{k}} y_{n_{k}}+\gamma_{n_{k}}, \\
K^{n_{k}} T y_{n_{k}-1} & =\alpha_{n_{k}} K^{n_{k}} y_{n_{k}}+K^{n_{k}} \gamma_{n_{k}}, \\
\left\langle T K^{n_{k}} y_{n_{k}-1}, K^{n_{k}} y_{n_{k}}-x_{0}\right\rangle & =\alpha_{n_{k}}\left\langle K^{n_{k}} y_{n_{k}}, K^{n_{k}} y_{n_{k}}-x_{0}\right\rangle \\
+\left\langle K^{n_{k}} \gamma_{n_{k}}, K^{n_{k}} y_{n_{k}}-x_{0}\right\rangle, & \\
\left\langle T K^{n_{k}} y_{n_{k}-1}, K^{n_{k}} y_{n_{k}}-x_{0}\right\rangle & =\alpha_{n_{k}}\left\langle K^{n_{k}} y_{n_{k}}, K^{n_{k}} y_{n_{k}}-x_{0}\right\rangle, \\
\lim _{k \rightarrow \infty}\left\langle T K^{n_{k}} y_{n_{k}-1}, K^{n_{k}} y_{n_{k}}-x_{0}\right\rangle & =0 .
\end{aligned}
$$

So,

$$
\left\langle T K z, y_{0}-x_{0}\right\rangle=0
$$

and

$$
\left\langle T z, K^{*}\left(y_{0}-x_{0}\right)\right\rangle=0
$$

Observe that the angle between $K^{n} y_{n}$ and $K^{n} y_{n}-x_{0}$ is bigger than $\frac{\pi}{2}$. So, $\left\|y_{0}\right\|^{2} \leq$ $\liminf \left\|K^{n_{k}} y_{n_{k}}\right\|^{2} \leq\left\|x_{0}\right\|^{2}-\epsilon^{2}$. That is, $y_{0}-x_{0} \neq 0$ and $K^{*}\left(y_{0}-x_{0}\right) \neq 0$. Note that $\overline{\{T z: T K=K T\}}$ is a closed subspace invariant under any operator commuting with $K$. This space is not the whole space because it is orthogonal to the nonzero vector $K^{*}\left(y_{0}-x_{0}\right)$.

Definition 1. A vector $x$ is called hypernoncyclic for an operator $T$ if the vector space $\{A x: A T=T A\}$, which is invariant under all operators commuting with $T$, is not dense. 
Theorem 5. Let $T$ be a quasinilpotent operator. Let $x_{0} \in H$. If for every $\delta_{1}>0$ and each $\delta_{2}$ with $0<\delta_{2}<\delta_{1}$ there exists a $\tau>0$ with

$$
\left\langle T^{n} y_{n}^{\epsilon}, T^{n} y_{n}^{\epsilon}-x_{0}\right\rangle \leq-\tau
$$

for all $n \geq 1$ and all $\epsilon$ in $\left[\delta_{2}, \delta_{1}\right]$, then $x_{0}$ is hypernoncyclic for $T$. In particular, $T$ has hyperinvariant subspaces.

Proof. We will first prove that for every $\delta_{1}$ and $\delta_{2}$ with $0<\delta_{2}<\delta_{1}$ there is a constant $K_{\delta_{1}, \delta_{2}}$ such that for all $n$

$$
\left\|y_{n}^{\delta_{2}}\right\| \leq K_{\delta_{1}, \delta_{2}} \cdot\left\|y_{n}^{\delta_{1}}\right\|
$$

The hypothesis of the theorem implies that for every $\epsilon$ in $\left[\delta_{2}, \delta_{1}\right]$ and every $n$, if the angle between $T^{n} y_{n}^{\epsilon}$ and $T^{n} y_{n}^{\epsilon}-x_{0}$ is $\theta_{n}^{\epsilon}$, then $\theta_{n}^{\epsilon}>\frac{\pi}{2}+\theta_{0}$ for some $\theta_{0}>0$. Consequently, $\sin \theta_{n}^{\epsilon}<t<1$ for all $n$, for all $\epsilon$ in $\left[\delta_{2}, \delta_{1}\right]$, and some $t$. For each $n$ there exists $\sigma_{n}^{\delta_{1}}$ such that $\left\|x_{0}-\left(1+\sigma_{n}^{\delta_{1}}\right) \cdot T^{n} y_{n}^{\delta_{1}}\right\|=\delta_{1} \cdot \sin \theta_{n}^{\delta_{1}}$. It can be easily seen that $\sigma_{n}^{\delta_{1}}<3 \delta_{1}$. Let $\epsilon_{n, 1}=\delta_{1} \cdot \sin \theta_{n}^{\delta_{1}}$. Then $\epsilon_{n, 1}<\delta_{1} \cdot t$ and

$$
\left\|y_{n}^{\epsilon_{n, 1}}\right\| \leq\left(1+3 \delta_{1}\right) \cdot\left\|y_{n}^{\delta_{1}}\right\| .
$$

If $\epsilon_{n, 1} \leq \delta_{2}$ let $\epsilon_{n, 2}=\epsilon_{n, 1}$. Otherwise, let $\epsilon_{n, 2}=\delta_{1} \cdot \sin \theta_{n}^{\delta_{1}} \cdot \sin \theta_{n}^{\epsilon_{n, 1}}$. Then, either $\epsilon_{n, 2}=\epsilon_{n, 1}$, or $\epsilon_{n, 2}<\delta_{1} \cdot t^{2}$ and

$$
\left\|y_{n}^{\epsilon_{n, 2}}\right\| \leq\left(1+3 \delta_{1}\right) \cdot\left(1+3 \delta_{1} \cdot t\right) \cdot\left\|y_{n}^{\delta_{1}}\right\| .
$$

Continuing inductively, for each $k \geq 1$ we define $\epsilon_{n, k}=\epsilon_{n,(k-1)}$ if $\epsilon_{n,(k-1)} \leq \delta_{2}$, and $\epsilon_{n, k}=\epsilon_{n,(k-1)} \cdot \sin \theta_{n}^{\epsilon_{n,(k-1)}}$, otherwise. Then, either $\epsilon_{n, k}=\epsilon_{n,(k-1)}$ or $\epsilon_{n, k} \leq$ $\delta_{1} \cdot t^{k-1}$, and for all $n$

$$
\left\|y_{n}^{\epsilon_{n, k}}\right\| \leq\left(1+3 \delta_{1}\right) \cdot\left(1+3 \delta_{1} \cdot t\right) \cdots\left(1+3 \delta_{1} \cdot t^{k-1}\right) \cdot\left\|y_{n}^{\delta_{1}}\right\| .
$$

There exists a $k_{0}$ such that $\delta_{1} \cdot t^{k_{0}} \leq \delta_{2}<\delta_{1} \cdot t^{k_{0}-1}$. Clearly, $\epsilon_{n, k_{0}+1}=\epsilon_{n, k_{0}}$ for all $n$. Let $K_{\delta_{1}, \delta_{2}}=\left(1+3 \delta_{1}\right) \cdot\left(1+3 \delta_{1} \cdot t\right) \cdots\left(1+3 \delta_{1} \cdot t^{k_{0}-1}\right)$. Then

$$
\left\|y_{n}^{\delta_{2}}\right\| \leq K_{\delta_{1}, \delta_{2}} \cdot\left\|y_{n}^{\delta_{1}}\right\| .
$$

Since $T$ is quasinilpotent, there is a sequence $\left(n_{k}\right)$ such that $\left\|y_{n_{k}}^{\delta_{1}}\right\| /\left\|y_{n_{k}-1}^{\delta_{1}}\right\| \rightarrow 0$ and $T^{n_{k}} y_{n_{k}}^{\delta_{1}} \rightarrow y_{0}$ weakly for some vector $y_{0}$. Let $A$ be any operator commuting with $T$. Without loss of generality we can assume that $\|T\| \leq 1$ and $\|A\| \leq 1$. Let $\alpha_{n_{k}}$ be scalars and $\gamma_{n_{k}}$ be vectors orthogonal to $y_{n_{k}}^{\delta_{1}}$ such that

$$
A y_{n_{k}-1}^{\delta_{2}}=\alpha_{n_{k}} \cdot y_{n_{k}}^{\delta_{1}}+\gamma_{n_{k}} .
$$

Then

$$
\left|\alpha_{n_{k}}\right|=\left|\left\langle A y_{n_{k}-1}^{\delta_{2}}, y_{n_{k}}^{\delta_{1}}\right\rangle\right| \cdot \frac{1}{\left\|y_{n_{k}}^{\delta_{1}}\right\|^{2}} \leq \frac{\left\|y_{n_{k}}^{\delta_{2}}\right\|}{\left\|y_{n_{k}-1}^{\delta_{1}}\right\|} \leq K_{\delta_{1}, \delta_{2}} \cdot \frac{\left\|y_{n_{k}}^{\delta_{1}}\right\|}{\left\|y_{n_{k}-1}^{\delta_{1}}\right\|} \rightarrow 0 .
$$

It follows that

$$
\left\langle A T^{n_{k}} y_{n_{k}-1}^{\delta_{2}}, T^{n_{k}} y_{n_{k}}^{\delta_{1}}-x_{0}\right\rangle \rightarrow 0 .
$$

Since, $A T^{n_{k}} y_{n_{k}-1}^{\delta_{2}}=A T x_{0}+\delta_{2} \cdot A T z_{n_{k}-1}$ for some $z_{n_{k}-1}$ with $\left\|z_{n_{k}-1}\right\|=1$,

$$
\left\langle A T x_{0}+\delta_{2} \cdot A T z_{n_{k}-1}, T^{n_{k}} y_{n_{k}}^{\delta_{1}}-x_{0}\right\rangle \rightarrow 0 \text {. }
$$


Consequently,

$$
\lim _{n \rightarrow 0}\left\|\left\langle A T x_{0}, T^{n_{k}} y_{n_{k}}^{\delta_{1}}-x_{0}\right\rangle\right\| \leq \delta_{2} .
$$

As the left-hand side of the above limit is independent of $\delta_{2}$,

$$
\lim _{n \rightarrow 0}\left\langle A T x_{0}, T^{n_{k}} y_{n_{k}}^{\delta_{1}}-x_{0}\right\rangle \rightarrow 0
$$

and

$$
\left\langle A T x_{0}, y-x_{0}\right\rangle=0
$$

In other words,

$$
\left\langle A x_{0}, T^{*}\left(y-x_{0}\right)\right\rangle=0
$$

Theorem 6. If $\sigma(T)=\{0\}$, then either " $A$ " or " $B$ " holds.

A. $T$ has hyperinvariant subspaces.

B. For every $x$ in $H$ with $\|x\|=1$, for every $\epsilon>0$, and for every $\gamma>\sqrt{2 \epsilon}$, there are $x^{\prime}$ with $\left\|x^{\prime}\right\|=1$ and $\epsilon^{\prime}$ with $0<\epsilon^{\prime}<\epsilon$ such that either $\mathbf{1}$ or $\mathbf{2}$ holds.

1. $\left\|x-x^{\prime}\right\|<\epsilon$.

2. For some subsequence $\left(n_{k}\right)$ of integers we have $T^{n_{k}} y_{n_{k}, x_{1}}^{\epsilon^{\prime}}=A_{n_{k}} x_{1}+S_{n_{k}}$, where $S_{n_{k}} \perp x_{1}$ and each weak limit of $S_{n_{k}}$ has norm less than $\gamma \cdot \epsilon^{\prime}$.

Proof. Assume " $B$ " does not hold for some unit vector $x_{0}$, some $\epsilon>0$ and some $\gamma>\sqrt{2 \epsilon}$. Since $T$ is quasinilpotent there is a subsequence $\left(y_{n_{k}, x_{0}}^{\epsilon}\right)$ of $\left(y_{n, x_{0}}^{\epsilon}\right)$ such that $\left\|y_{n_{k}, x_{0}}^{\epsilon}\right\| /\left\|y_{n_{k}, x_{0}}^{\epsilon}\right\| \rightarrow 0$. Put $T^{n_{k}} y_{n_{k}, x_{0}}^{\epsilon}=A x_{0}+w_{0}+S_{n_{k}}$, where $S_{n_{k}} \rightarrow 0$ weakly. Since " $B$ " does not hold, $\left\|w_{0}\right\|>\gamma \cdot \epsilon$. It follows that $\left\|S_{n_{k}}\right\|<\left(1-\frac{\gamma^{2}}{2}\right) \cdot \epsilon$. We get

$$
\left\|y_{n_{k}, x_{0}}^{\left(1-\left(\gamma^{2} / 2\right)\right) \epsilon}\right\| \leq\left\|y_{n_{k}, x_{0}}^{\epsilon}\right\| .
$$

Let $x_{1}=A x_{0}+w_{0}$. We have $\left\|x_{1}\right\| \geq 1-\epsilon$. So, after normalization we get (with $\left.x_{1}^{\prime}=\frac{x_{1}}{\left\|x_{1}\right\|}\right)$

$$
\left\|y_{n_{k}, x_{1}^{\prime}}^{\frac{1-\left(\gamma^{2} / 2\right)}{1-\epsilon}} \epsilon \leq \frac{1}{1-\epsilon} \cdot\right\| y_{n_{k}, x_{0}}^{\epsilon} \|
$$

We now start the process over with $x_{1}^{\prime}$ instead of $x_{0}, e_{1}^{\prime}=\frac{1-\left(\gamma^{2} / 2\right)}{1-\epsilon} \epsilon$ instead of $\epsilon$. Either we have " $B$ " or we get $x_{2}^{\prime}$ with

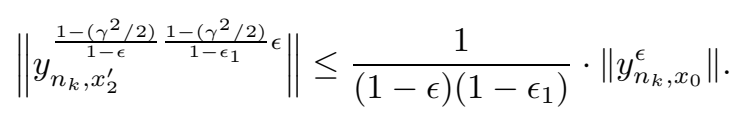

We see that the sequence $x_{0}, x_{1}^{\prime}, x_{2}^{\prime}, \ldots$ converges, since $\left\|x_{n}^{\prime}-x_{n-1}^{\prime}\right\|<\left(\frac{1-\gamma^{2}}{1-\epsilon}\right)^{n}$. Since $\epsilon_{n}<\left(\frac{1-\left(\gamma^{2} / 2\right)}{1-\epsilon}\right)^{n} \cdot \epsilon$, either we have " $B^{\prime \prime}$ for some $n$, or with $x=\lim x_{n}^{\prime}$ we will have $x=T^{n_{k}} w_{n_{k}}$ with $\left\|w_{n_{k}}\right\|<K \cdot\left\|y_{n_{k}, x_{0}}^{\epsilon}\right\|$ for all $k$, where $K=\frac{1}{(1-\epsilon) \prod_{n}\left(1-\epsilon_{n}\right)}$. An argument similar to the proof of Theorem 4 implies that $x$ is hypernoncyclic for $T$.

Theorem 7. Assume that $T$ is normal, has a cyclic vector and has dense range $\mathcal{R}(T)$. Assume $x_{0} \notin \mathcal{R}(T)$. Then, for any $x_{0}$, the sequence $\left(T^{n} y_{n}^{\epsilon}\right)$ converges in norm to a hypernoncyclic vector. 
Proof. We can assume that $0 \in \sigma(T)$. Represent $T$ as multiplication by $z$ on $L^{2}(\mu)$, where $\mu$ is a regular Borel measure on $\sigma(T)$. Let $x_{0}=f$. We have the following lemma, whose proof is obvious.

Lemma 2. Given $\epsilon>0$, there are $a \delta>0$ and $0<\alpha<1$ such that if $g(z)=0$ for $|z|<\delta, g(z)=\alpha f(z)$ for $|z|=\delta$ and $g(z)=f(z)$ for $|z|>\delta$, then $\|f-g\|=\epsilon$. If $\mu\{z:|z|=\delta\}=0$, then $\alpha$ is arbitrary.

We have $T^{n} y_{n}^{\epsilon}=\delta_{n} T^{n}\left(I-\delta_{n} T^{* n} T^{n}\right)^{-1} T^{* n} x_{0}=\frac{\delta_{n}|z|^{2 n}}{1+\delta_{n}|z|^{2 n}} \cdot f(z)$. Let $\delta$ and $\alpha$ be as in the above lemma. We will show that

$$
\frac{\delta_{n}|z|^{2 n}}{1-\delta_{n}|z|^{2 n}} \rightarrow 0 \quad \forall|z|<\delta
$$

Assume not. Then, there are $\delta^{\prime}<\delta$ and $\gamma>0$ such that $\frac{\delta_{n}\left|\delta^{\prime}\right|^{2 n}}{1+\delta_{n}\left|\delta^{\prime}\right|^{2 n}}>\gamma$ for infinitely many $n$, say for the sequence $n_{\nu}$. Then for all $z$ with $|z|>\delta^{\prime}$ we have $\delta_{n_{\nu}}|z|^{2 n_{\nu}} \rightarrow \infty$ as $\nu \rightarrow \infty$. So, $\frac{\delta_{n_{\nu}}|z|^{2 n_{\nu}}}{1-\delta_{\nu}|z|^{2 n_{\nu}}} \rightarrow 1$ for all $z$ with $|z|>\delta^{\prime}$. But by Lemma 2 and since $0 \leq \frac{\delta_{n_{\nu}}|z|^{2 n_{\nu}}}{1-\delta_{\nu}|z|^{2 n_{\nu}}}<1$ for all $z$, we get $\left\|\frac{\delta_{n_{\nu}}|z|^{2 n_{\nu}}}{1-\delta_{n_{\nu}}|z|^{2 n_{\nu}}} \cdot f-f\right\|<\epsilon$ for $\nu$ large enough. This contradiction gives (1), and in the same way we get

$$
\frac{\delta_{n}|z|^{2 n}}{1-\delta_{n}|z|^{2 n}} \rightarrow 1 \quad \forall|z|>\delta
$$

Now if $\mu\{z:|z|=\delta\}>0$, then (1), (2), and Lemma 2 give $\frac{\delta_{n}|z|^{2 n}}{1-\delta_{n}|z|^{2 n}} \rightarrow \alpha$ $\forall|z|=\delta$, as otherwise $\left\|\frac{\delta_{n}|z|^{2 n}}{1-\delta_{n}|z|^{2 n}} \cdot f-f\right\|=\epsilon$ would not hold for $n$ large enough. Hence, $T^{n} y_{n} \rightarrow h$ in norm, where $h(z)=0$ if $|z|<\delta, h(z)=\alpha \cdot f(z)$ if $|z|=\delta$, and $h(z)=f(z)$ if $|z|>\delta$. Let $S_{\delta}=\{z:|z| \geq \delta\}$, and let $\mathcal{K}_{S_{\delta}}$ be the characteristic function of $S_{\delta}$. Then $h \cdot L^{2}(\mu) \subset \mathcal{K}_{S_{\delta}} \cdot L^{2}(\mu)$, and $\mathcal{K}_{S_{\delta}} \cdot L^{2}(\mu)$ is a hyperinvariant subspace for $T$. So, $h$ is a hypernoncyclic vector for $T$.

Remark 2. A. Note that in the proof of Theorem 7 , as $\epsilon \rightarrow 0, \delta \rightarrow 0$. So, as $\epsilon \rightarrow 0$, $K_{S_{\delta}} \rightarrow 1_{\sigma(T)}$. It follows that if $x_{0}=1_{\sigma(T)}$, then as $\epsilon \rightarrow 0$, the hyperinvariant subspaces corresponding to $\epsilon$ grow to $H$.

B. The proof of Theorem 4 can be written in terms of $\left(v_{n}\right)$.

C. From the proof of Theorem 4 it is clear that if $T$ is quasinilpotent and either $\left(v_{n, x_{0}}^{\epsilon}\right)$ or $\left(T^{n} y_{n, x_{0}}^{\epsilon}\right)$ converges in norm for some $x_{0}$ and some $\epsilon$ with $0<\epsilon<\left\|x_{0}\right\|$, then $T$ has hyperinvariant subspaces.

D. Suppose $T$ is either a compact operator or a normal operator such that $\mathcal{R}(T)$ is dense and is not the whole space. Then, the set of hypernoncyclic vectors of $T$ is dense. In particular, if an operator $A$ commutes with $T$, then $A$ has a dense set of noncyclic vectors. In other words, any operator with an open set of cyclic vectors does not commute with $T$.

\section{Operators of Class $\mathcal{R}$}

Suppose $T: H \rightarrow H$ is a noninvertible bounded linear operator with dense range and $x_{0} \notin \mathcal{R}(T)$. In the previous section it was shown that if $T$ is normal then $\left(T^{n} y_{n, x_{0}}^{\epsilon}\right)$ converges in norm to a hypernoncyclic vector, that if $T$ is quasinilpotent and $\left(T^{n} y_{n, x_{0}}^{\epsilon}\right)$ converges in norm, then the norm limit is hypernoncyclic, and that if $T$ is compact, then some weak limit of $\left(T^{n} y_{n, x_{0}}^{\epsilon}\right)$ is hypernoncyclic. This shows that the norm convergence of $\left(T^{n} y_{n, x_{0}}^{\epsilon}\right)$ is an important issue, and raises the following 
question. Suppose $T$ is a nonsurjective bounded linear operator with dense range. Suppose the set $\mathcal{N}=\left\{x_{0}: x_{0} \notin \mathcal{R}(T)\right.$ and $T^{n} y_{n, x_{0}}^{\epsilon} \rightarrow y_{x_{0}}^{\epsilon}$ in norm $\} \neq \emptyset$. Is it true that for some $x_{0} \in \mathcal{N}$ the norm limit $y_{x_{0}}^{\epsilon}$ of $\left(T^{n} y_{n, x_{0}}^{\epsilon}\right)$ is noncyclic? In this section and the next we work on the norm convergence of $\left(T^{n} y_{n, x_{0}}^{\epsilon}\right)$ and $\left(v_{n, x_{0}}^{\epsilon}\right)$. Remark 7 concerns the question we just posed.

Definition 2. Let $T: X \rightarrow X$ be a continuous linear operator. A vector $x$ in $X$ is said to be quasimaximal for $T$ if $\frac{\left\|T^{n} x\right\|}{\left\|T^{n}\right\|} \geq \delta_{x}$ for some $\delta_{x}>0$.

Lemma 3. Let $T: H \rightarrow H$ be any bounded linear operator, $x_{0} \in H,\left\|x_{0}\right\|=1$ and $0<\epsilon<\left\|x_{0}\right\|$. Let $\delta_{n, x_{0}}^{\epsilon}$ be the negative constant appearing in the orthogonality equation $v_{n, x_{0}}^{\epsilon}-x_{0}=\delta_{n, x_{0}}^{\epsilon} T^{* n} T^{n} v_{n, x_{0}}^{\epsilon}$. Then, the following hold true.

a. $\epsilon<-\delta_{n, x_{0}}^{\epsilon}\left\|T^{n}\right\|^{2} \quad \forall n \geq 0$.

b. The sequence $\left(-\delta_{n, x_{0}}^{\epsilon}\left\|T^{n}\right\|^{2}\right)$ is bounded from above if and only if there exists a $\delta$ such that $0<\delta<\frac{\left\|T^{n} x\right\|}{\left\|T^{n}\right\|} \quad \forall x \in \overline{B\left(x_{0}, \epsilon\right)} \forall n$.

c. If $x_{0}$ is quasimaximal for $T$, then there exist $\epsilon>0$ and $\delta_{\epsilon}>0$ such that $\| \frac{\left\|T^{n} x\right\|}{\left\|T^{n}\right\|} \geq \delta_{\epsilon}>0 \quad \forall n$ and $\forall x \in \overline{B\left(x_{0}, \epsilon\right)}$. That is, the set of all quazimaximal vectors is open. (Here $T$ can be assumed to be an operator from $X$ to $X$.)

d. If $x_{0}$ is quasimaximal, then there exists an $\epsilon>0$ such that $\left(-\delta_{n, x_{0}}^{\epsilon}\left\|T^{n}\right\|^{2}\right)$ is bounded from above.

Proof. (a) Let $v_{n}=v_{n, x_{0}}^{\epsilon}$. From the orthogonality equation we have

$$
\epsilon=-\delta_{n, x_{0}}^{\epsilon}\left\|T^{* n} T^{n} v_{n}\right\| \leq-\delta_{n, x_{0}}^{\epsilon}\left\|T^{n}\right\|^{2}\left\|v_{n}\right\| \leq-\delta_{n, x_{0}}^{\epsilon}\left\|T^{n}\right\|^{2} .
$$

(b) Suppose $0<\delta<\frac{\left\|T^{n} x\right\|}{\left\|T^{n}\right\|} \forall n \forall x \in \overline{B\left(x_{0}, \epsilon\right)}$. Then,

$$
\delta^{2} \leq \frac{\left\|T^{n} v_{n}\right\|^{2}}{\left\|T^{n}\right\|^{2}} \leq \frac{\left\|T^{* n} T^{n} v_{n}\right\|}{\left\|T^{n}\right\|^{2}}=\frac{\epsilon}{-\delta_{n, x_{0}}^{\epsilon}\left\|T^{n}\right\|^{2}} .
$$

So, $-\delta_{n, x_{0}}^{\epsilon}\left\|T^{n}\right\|^{2} \leq \frac{\epsilon}{\delta^{2}}$. Conversely, suppose the sequence $\left(-\delta_{n, x_{0}}^{\epsilon}\left\|T^{n}\right\|^{2}\right)$ is bounded. From equation (1) we have

$$
-\delta_{n, x_{0}}^{\epsilon}\left\|T^{n}\right\|^{2}=-\delta_{n, x_{0}}^{\epsilon}\left\|T^{* n} T^{n}\right\|=\frac{\epsilon\left\|T^{* n} T^{n}\right\|}{\left\|T^{* n} T^{n} v_{n}\right\|} \leq M .
$$

So, $\frac{\epsilon\left\|T^{* n} T^{n}\right\|}{M} \leq\left\|T^{* n} T^{n} v_{n}\right\| \leq\left\|T^{* n}\right\|\left\|T^{n} v_{n}\right\|$. Hence,

$$
\frac{\epsilon}{M} \leq \frac{\left\|T^{n} v_{n}\right\|}{\left\|T^{n}\right\|} \leq \frac{\left\|T^{n} x\right\|}{\left\|T^{n}\right\|} \quad \forall n \geq 0, \forall x \in \overline{B\left(x_{0}, \epsilon\right)} .
$$

(c) Let $0<\rho \leq \frac{\left\|T^{n} x_{0}\right\|}{\left\|T^{n}\right\|} \forall n \geq 0$. Let $0<\epsilon<\rho$. Then,

$$
\left\|x_{0}-y\right\|<\epsilon \Longrightarrow \frac{\left\|T^{n} x_{0}\right\|}{\left\|T^{n}\right\|}-\frac{\left\|T^{n} y\right\|}{\left\|T^{n}\right\|} \leq\left\|\frac{T^{n} x_{0}}{\left\|T^{n}\right\|}-\frac{T^{n} y}{\left\|T^{n}\right\|}\right\|<\epsilon .
$$

That is, $\rho-\epsilon \leq \frac{\left\|T^{n} y\right\|}{\left\|T^{n}\right\|}$. Let $\delta=\rho-\epsilon$.

(d) is clear from (b) and (c).

Remark 3. a. From Lemma 3 it follows that if $T$ is quasinilpotent injective and with dense range, then for any $x_{0}$ with $\left\|x_{0}\right\|=1,-\delta_{n, x_{0}}^{\epsilon}\left\|T^{n}\right\|^{2} \rightarrow \infty$.

b. If $x$ is nonquasimaximal for $T$, then the space $\{A x: A T=T A\}$ consists of nonquasimaximal vectors. Consequently, if $T$ has a quasimaximal and a nonzero nonquasimaximal vector, then $T$ has hyperinvariant subspaces. 
c. Suppose $T$ is quasinilpotent. Then, $\frac{\| T^{n}{ }^{n}+1}{\left\|T^{n}\right\|} \rightarrow 0$ for some subsequence. So, for any vector $x$ the vector $T x$ cannot be quasimaximal for $T$. Thus if $T$ has a quasimaximal vector $x$, then $T x$ is hypernoncyclic. It is easy to see that for any dense range operator $A$, if a vector $A y$ is hypernoncyclic (cyclic) for $A$, then $y$ is itself hypernoncyclic (cyclic) for $A$. Thus, if $T$ is quasinilpotent with dense range and with a quasimaximal vector, then all vectors are hypernoncyclic for $T$.

Definition 3. A bounded linear operator $T: X \rightarrow X$ is said to be of class $\mathcal{R}$ if each nonzero vector is quaisimaximal for $T$.

Definition 4. A sequence $\left(a_{n}\right)$ of real numbers is called admissible for a bounded linear operator $T$ if there exist constants $k>0$ and $K<\infty$ such that for all $n \geq 1$

$$
k \cdot\left\|T^{n}\right\| \leq a_{n} \leq K \cdot\left\|T^{n}\right\| .
$$

Remark 4. a. Suppose $\left(a_{n}\right)$ is a sequence such that for each nonzero vector $x$ there exist $\delta_{x}$ and $\tau_{x}$ with

$$
0<\delta_{x} \leq \frac{\left\|T^{n} x\right\|}{a_{n}} \leq \tau_{x}<\infty .
$$

Then there exist constants $k$ and $K$ such that

$$
k \cdot\left\|T^{n}\right\| \leq a_{n} \leq K \cdot\left\|T^{n}\right\| .
$$

Consequently, if $(*)$ holds for each nonzero $x$, then $T$ is of class $\mathcal{R}$. Conversely, if $T$ is of class $\mathcal{R}$, and $\left(a_{n}\right)$ is an admissible sequence, then $(*)$ holds for each nonzero vector $x$.

b. If $T$ is of class $\mathcal{R}$, then $r(T)>0$. If $\left(a_{n}\right)$ is an admissible sequence for $T$ and $\frac{a_{n+1}}{a_{n}} \rightarrow t$, then $t=r(T)$. Recall that a $C_{1}$-contraction is an operator $T: X \rightarrow X$ such that $\left\|T^{n}\right\|=1$ for all $n$ and for each $x \neq 0$ there exists $\delta_{x}>0$ such that $\delta_{x}<\left\|T^{n} x\right\|$ for all $n$. Clearly, a $C_{1}$-contraction is of class $\mathcal{R}$. Recall that an operator $T$ is said to be power bounded if there exist $\delta>0$ and $M<\infty$ such that $\delta<\left\|T^{n}\right\|<M$ for all $n$. Example 1 in the next section shows that an operator of class $\mathcal{R}$ is not necessarily power bounded (even if $r(T)=1$ ).

Proof. (a) Since $\frac{\left\|T^{n} x\right\|}{a_{n}} \leq \tau_{x}<\infty$ for each $x$ in $X$, the uniform boundedness principle implies that $\frac{\left\|T^{n}\right\|}{a_{n}} \leq M$ for some $M<\infty$. Let $k=\frac{1}{M}$. Now if there is no $K$ such that $a_{n} \leq K \cdot\left\|T^{n}\right\|$, then $\frac{a_{n}}{\left\|T^{n}\right\|} \rightarrow \infty$. So, $\frac{\left\|T^{n}\right\|}{a_{n}} \rightarrow 0$. Hence, $\frac{\left\|T^{n} x\right\|}{a_{n}} \rightarrow 0$. This contradicts the hypothesis. Thus, $(*)$ implies $(* *)$. The rest of the conclusion can be seen easily.

Let $l^{\infty}$ denote the Banach space of all bounded sequences of complex numbers. Recall (see [3]) that there are linear functionals $\mathcal{F}: l^{\infty} \rightarrow \mathcal{C}$ and $\mathcal{G}: l^{\infty} \rightarrow \mathcal{C}$ with the following properties.

(1) $\mathcal{F}\left(\left(\xi_{n}\right)\right)=$ limit of a subsequence of $\left(\xi_{n}\right)$.

(2) $\mathcal{F}\left(\left(\xi_{n}\right)\right) \leq \mathcal{F}\left(\left(\xi_{n}^{\prime}\right)\right)$ if $\xi_{n}, \xi_{n}^{\prime}$ are real and $\xi_{n} \leq \xi_{n}^{\prime} \forall n \geq 1$.

$\left(1^{\prime}\right) \mathcal{G}\left(\left(\xi_{n}\right)\right)=$ limit of a subsequence of $\left(\frac{\xi_{1}+\ldots+\xi_{n}}{n}\right)$.

$\left(2^{\prime}\right) \mathcal{G}\left(\left(\xi_{n}\right)\right) \leq \mathcal{G}\left(\left(\xi_{n}^{\prime}\right)\right)$ if $\xi_{n}, \xi_{n}^{\prime}$ are real and $\xi_{n} \leq \xi_{n}^{\prime} \forall n \geq 1$.

$\left(3^{\prime}\right) \mathcal{G}\left(\left(\xi_{n}\right)\right)=\mathcal{G}\left(\left(\xi_{n+1}\right)\right)$. That is, $\mathcal{G}$ is invariant under the shift.

Recall that a linear functional $\mathcal{F}$ or $\mathcal{G}$ as above is a Banach limit (see [3]). In this article we will consider Banach limits that satisfy either 1 and 2 or $1^{\prime}-3^{\prime}$. 
Remark 5. a. Suppose $T: H \rightarrow H$ is of class $\mathcal{R},\left(a_{n}\right)$ is an admissible sequence for $T$, and $\mathcal{F}$ is any Banach limit. Then, the function $[\cdot, \cdot]: H \rightarrow H$ defined by

$$
[x, y]_{a_{n}}=\mathcal{F}\left\langle\frac{T^{n} x}{a_{n}}, \frac{T^{n} y}{a_{n}}\right\rangle
$$

is an inner product on $H$ and it defines a Hilbert norm $\|\cdot\|_{\mathcal{F}, a_{n}}$ on $H$ by $\|x\|_{\mathcal{F}, a_{n}}^{2}=$ $\mathcal{F}\left(\frac{\left\|T^{n} x\right\|^{2}}{a_{n}^{2}}\right)$. The norms $\|\cdot\|_{\mathcal{F}, a_{n}}$ and $\|\cdot\|_{\mathcal{F},\left\|T^{n}\right\|}$ are equivalent.

b. Suppose $T: X \rightarrow X$ is of class $\mathcal{R}$ and $\left(a_{n}\right)$ is an admissible sequence for $T$. Let $\mathcal{F}$ be any Banach limit. Define $\|x\|_{\mathcal{F}, a_{n}}=\mathcal{F}\left(\left(\frac{\left\|T^{n} x\right\|}{a_{n}}\right)\right)$ for all $x \in X$. Then, $\|\cdot\|_{\mathcal{F}, a_{n}}$ is a norm on $X$. The norms $\|\cdot\|_{\mathcal{F}, a_{n}}$ and $\|\cdot\|_{\mathcal{F},\left\|T^{n}\right\|}$ are equivalent.

c. Suppose $T$ is of class $\mathcal{R},\left(a_{n}\right)$ is an admissible sequence for $T$ and $\mathcal{G}$ is a Banach limit invariant under the shift (i.e. $\mathcal{G}\left(x_{n}\right)=\mathcal{G}\left(x_{n+1}\right)$ ). Then, $T$ extends uniquely to a bounded linear operator $\tilde{T}$ from the completion $\tilde{X}$ to itself. If $\lim \frac{a_{n+1}}{a_{n}}=r$, then $A=\frac{T}{r}:\left(X,\|\|_{\mathcal{G}, a_{n}}\right) \rightarrow\left(X,\|\cdot\|_{\mathcal{G}, a_{n}}\right)$ is an isometry, which obviously extends to an isometry $\tilde{A}$ from the completion $\tilde{X}$ of $X$ onto itself. In particular, if $T$ is power bounded, $\left(a_{n}\right)$ is any admissible sequence for $T$ with $\frac{a_{n+1}}{a_{n}} \rightarrow 1$ (e.g. $a_{n}=1 \forall n$ ), and $\mathcal{F}$ is a Banach limit invariant under the shift, then $\tilde{T}:\left(\tilde{X},\|\cdot\|_{\mathcal{F}, a_{n}}\right) \rightarrow\left(\tilde{X},\|\cdot\|_{\mathcal{F}, a_{n}}\right)$ is an isometry. The case when $T$ is power bounded has already been considered by C. Foias and B. Sz.-Nagy [4].

Proof. (a) It is easy to see that $\|\cdot\|_{\mathcal{F}, a_{n}}$ is a Hilbert norm. For the equivalence of $\|\cdot\|_{\mathcal{F}, a_{n}}$ and $\|\cdot\|_{\mathcal{F},\left\|T^{n}\right\|}$ note that there exist constants $m$ and $M$ with

$$
m^{2} \cdot \frac{\left\|T^{n} x\right\|^{2}}{\left\|T^{n}\right\|^{2}} \leq \frac{\left\|T^{n} x\right\|^{2}}{a_{n}^{2}} \leq M^{2} \cdot \frac{\left\|T^{n} x\right\|^{2}}{\left\|T^{n}\right\|^{2}} .
$$

(b) This is similar to (a).

(c) Suppose $\|\cdot\| \mathcal{F} a_{n}$ is defined as in (a).

$$
\|T x\|_{a_{n}}^{2}=\mathcal{F}\left(\frac{\left\|T^{n+1} x\right\|^{2}}{a_{n}{ }^{2}}\right)=\mathcal{F}\left(\frac{\left\|T^{n+1} x\right\|^{2}}{a_{n+1}{ }^{2}} \cdot \frac{a_{n+1}{ }^{2}}{a_{n}{ }^{2}}\right) .
$$

For any $\epsilon>0$ we have

$$
\mathcal{F}\left(\frac{\left\|T^{n+1} x\right\|^{2}}{a_{n+1}{ }^{2}} \cdot(r-\epsilon)^{2}\right) \leq \mathcal{F}\left(\frac{\left\|T^{n+1} x\right\|^{2}}{a_{n+1}{ }^{2}} \cdot \frac{a_{n+1}^{2}}{a_{n}{ }^{2}}\right) \leq \mathcal{F}\left(\frac{\left\|T^{n+1} x\right\|^{2}}{a_{n+1}{ }^{2}} \cdot(r+\epsilon)^{2}\right) .
$$

It follows that

$$
\mathcal{F}\left(\frac{\left\|T^{n+1} x\right\|^{2}}{a_{n+1}{ }^{2}} \cdot \frac{a_{n+1}^{2}}{a_{n}{ }^{2}}\right)=r^{2} \cdot \mathcal{F}\left(\frac{\left\|T^{n+1} x\right\|^{2}}{a_{n+1}{ }^{2}}\right)=r^{2} \cdot\|x\|_{a_{n}}^{2} .
$$

The proof is similar if $\|\cdot\|_{\mathcal{F}, a_{n}}$ is defined as in (b).

Remark 6. A result of C. Foias and B. Sz.-Nagy [4] states that if $T: H \rightarrow H$ is power bounded, and for each $x \neq 0$ there exists $\delta_{x}>0$ such that $\delta_{x}<\left\|T^{n} x\right\|$ and $\delta_{x}<\left\|T^{* n} x\right\| \forall n$, then $T$ is quasisimilar to a unitary operator, and hence it has hyperinvariant subspaces. This result extends (with a similar proof) to a Hilbert space operator of class $\mathcal{R}$ as follows. If $T$ and $T^{*}$ both are of class $\mathcal{R}$ and if there is an admissible sequence $\left(a_{n}\right)$ such that $\frac{a_{n+1}}{a_{n}} \rightarrow r=r(T)$, then $\frac{T}{r}$ is quasisimilar to a unitary operator. Hence, $T$ has hyperinvariant subspaces.

Notation. Let $T: X \rightarrow X$ be a bounded linear operator with dense range. Let $X_{0}=\left\{x \in X: T^{-n} x\right.$ exists $\left.\forall n\right\}$. Recall that $X_{0}$ is dense (see [1]). 
Lemma 4. Suppose $T: X \rightarrow X$ is of class $\mathcal{R},\left(a_{n}\right)$ is a sequence such that for some positive constants $k$ and $K, k \cdot\left\|T^{n}\right\| \leq a_{n} \leq K \cdot\left\|T^{n}\right\|$ for all $n \geq 0$ and $\frac{a_{n+1}}{a_{n}} \rightarrow r$. Then, the following hold true.

a. $F=\left\{g \in(X,\|\cdot\|)^{\prime}: T^{*^{-n}} g\right.$ exists $\forall n$, and $\left.\sup _{n}\left\|T^{*^{-n}} g\right\| \cdot r^{n}<\infty\right\} \neq\{0\}$.

b. For any $g \in F$ define $|g|=\sup _{n}\left\|T^{*^{-n}} g\right\| \cdot r^{n}$, then, $|\cdot|$ is a norm on $F$.

c. Let $x \in X_{0}$.

1. If $\mathcal{F}$ is any Banach limit for which $\mathcal{F}\left(\left(\xi_{n}\right)\right)=$ the limit of some subsequence of $\left(\xi_{n}\right)$, then, $\left\|T^{-m} x\right\|_{\mathcal{F}, a_{n}} \leq \frac{r^{-m}}{k} \cdot\|x\|$.

2. Suppose $\frac{T}{r}$ is power bounded and $\mathcal{F}$ is any Banach limit. Then, there exists a constant $K^{\prime}$ such that $\left\|T^{-m} x\right\|_{\mathcal{F}, a_{n}} \leq K^{\prime} \cdot r^{-m} \cdot\|x\|$.

Consequently, $T^{-m}:\left(X_{0},\|\cdot\|\right) \rightarrow\left(\tilde{X},\|\cdot\|_{\mathcal{F}, a_{n}}\right)$ is continuous.

d. Let $\mathcal{F}$ be any Banach limit such that $\left\|T^{-m} x\right\|_{\mathcal{F}, a_{n}} \leq K^{\prime} \cdot r^{-m} \cdot\|x\|$ for all $x \in X_{0}$ and some $K^{\prime}$. Then, for any $\tilde{f} \in\left(\tilde{X},\|\cdot\|_{\mathcal{F}, a_{n}}\right)^{\prime}, T^{*^{-m}}\left(\left.\tilde{f}\right|_{X}\right)$ exists for all $m$.

e. Let $\mathcal{F}$ be as in $\mathbf{d}$. Then, for any $\tilde{f} \in\left(\tilde{X},\|\cdot\|_{\mathcal{F}, a_{n}}\right)^{\prime}$ we have $f=\left.\tilde{f}\right|_{X} \in F$ and $|f| \leq K^{\prime} \cdot\|\tilde{f}\|_{\mathcal{F}, a_{n}}$.

f. Suppose $\frac{T}{r}$ is power bounded. Suppose $\mathcal{F}$ is any Banach limit. Suppose $F$ is $\|\cdot\|$-dense in $(X,\|\cdot\|)^{\prime}$. Then, the mapping $J:(F,|\cdot|) \rightarrow\left(\tilde{X},\|\cdot\|_{\mathcal{F}, a_{n}}\right)^{\prime}$ defined by $J(f)=\tilde{f}$ is an isomorphism, where $\tilde{f}$ denotes the unique extension of $f$ to all of $\tilde{X}$. That is, there exist $K^{\prime}$ and $K^{\prime \prime}$ such that $K^{\prime \prime} \cdot\|\tilde{f}\|_{\mathcal{F}, a_{n}} \leq|f| \leq$ $K^{\prime} \cdot\|\tilde{f}\|_{\mathcal{F}, a_{n}}$. If $\mathcal{F}$ is invariant under the shift, then $T$ extends to a unique bounded linear operator $\tilde{T}$ from $\left(\tilde{X},\|\cdot\|_{\mathcal{F}, a_{n}}\right)$ to itself.

1. For any $f$ in $F,(\tilde{T})^{*} \tilde{f}=\left(T^{*} f \tilde{)}=J\left(T^{*} f\right)\right.$.

2. Suppose $A$ is any subspace of $F$. Then, $A$ is invariant under $T^{*}$ iff $J A$ is invariant under $(\tilde{T})^{*}$.

Proof. a. Let $x \neq 0$ and $0<\delta_{x}<\frac{\left\|T^{n} x\right\|}{a_{n}}$. Let $\left(f_{n}\right)$ be a sequence in $(X,\|\cdot\|)$ such that $\left\|f_{n}\right\|=1$ and $\left|f_{n}\left(\frac{T^{n} x}{a_{n}}\right)\right|>\delta_{x}$. Then, $\left|\frac{T^{* n} f_{n}}{a_{n}}(x)\right|>\delta_{x}$. That is, $\frac{T^{* n} f_{n}}{a_{n}} \not$ 0 in the $w^{*}$-topology. Since this sequence is bounded, it has a $w^{*}$-convergent subsequence, say $\frac{T^{* n 1} f_{n 1}}{a_{n 1}} \rightarrow g_{1}$ in $(X,\|\cdot\|)^{\prime}$. As $\left(\frac{\left\|T^{* n 1-1} f_{n 1}\right\|}{a_{n 1-1}}\right)$ is bounded (by $\left.\frac{1}{k}\right)$, it has a convergent subsequence, say $\frac{T^{* n 2-1} f_{n 2}}{a_{n 2-1}} \rightarrow g_{2}$. Note that

$$
\frac{T^{* n 2} f_{n 2}}{a_{n 2}}=\left(\frac{a_{n 2-1}}{a_{n 2}}\right) \cdot T^{*}\left(\frac{T^{*^{n 2-1}} f_{n 2}}{a_{n 2-1}}\right) .
$$

It follows that $g_{1}=\frac{1}{r} T^{*} g_{2}$. That is, $T^{*} g_{2}=r \cdot g_{1}$. Applying this argument repeatedly and passing to the diagonal subsequence, we get a sequence $\left(n_{k}\right)$ of natural numbers such that $\frac{T^{* n_{k}-m} f_{n_{k}}}{a_{n_{k}-m}} \rightarrow g_{m}$ in the $w^{*}$-topology. Since

$$
\frac{T^{*^{n_{k}}} f_{n_{k}}}{a_{n_{k}}}=\left(\frac{a_{n_{k}-m}}{a_{n_{k}}}\right) \cdot T^{* m}\left(\frac{T^{*^{n_{k}-m}} f_{n_{k}}}{a_{n_{k}-m}}\right),
$$

it follows that $T^{* m} g_{m}=r^{m} \cdot g_{1}$. That is, $\left\|T^{*^{-m}} g_{1}\right\| \cdot r^{m}=\left\|g_{m}\right\|$. Note that $\left\|g_{m}\right\| \leq \frac{1}{k}$. This means that $g_{1} \in F$.

b. This is obvious. 
c. 1. There is some subsequence $\left(n_{k}\right)$ of natural numbers such that

$$
\begin{aligned}
\left\|T^{-m} x\right\|_{a_{n}, \mathcal{F}} & =\lim _{k \rightarrow \infty} \frac{\left\|T^{n_{k}-m} x\right\|}{a_{n_{k}}}=\lim _{k \rightarrow \infty} \frac{\left\|T^{n_{k}-m} x\right\|}{a_{n_{k}-m}} \cdot\left(\frac{a_{n_{k}-m}}{a_{n_{k}}}\right) \\
& \leq \frac{1}{k} \cdot r^{-m} \cdot\|x\| .
\end{aligned}
$$

2. Since $\frac{T}{r}$ is power bounded, there exist $c>0$ and $C<\infty$ such that $c \cdot\left\|T^{n}\right\| \leq$ $\left\|T^{n}\right\| \leq C \cdot\left\|T^{n}\right\| \forall n$. In view of (1) we can assume that $\mathcal{F}\left(\left(\xi_{n}\right)\right)=$ the limit of a subsequence of $\left(\frac{\xi_{1}+\cdots+\xi_{n}}{n}\right)$. Let $x \in X_{0} .\left\|T^{*^{-m}} x\right\|_{\mathcal{F}, a_{n}}=$ limit of a subsequence of $\sum_{i=1}^{j-m} \frac{\left\|T^{i-m} x\right\|}{j \cdot a_{i}}=$ the limit of a subsequence of $\sum_{i=m}^{j-m} \frac{\left\|T^{i-m} x\right\|}{j \cdot a_{i}}$. Each term

$$
\sum_{i=m}^{j-m} \frac{\left\|T^{i-m} x\right\|}{j \cdot a_{i}}=\sum_{i=m}^{j-m} \frac{\left\|T^{i-m} x\right\|}{j \cdot a_{i-m}} \cdot \frac{a_{i-m}}{a_{i}} \leq \frac{1}{k} \cdot \frac{(j-2 m)}{j} \cdot \frac{C}{c} \cdot r^{-m} \cdot\|x\| .
$$

Let $K^{\prime}=\frac{C}{k \cdot c}$.

d. For any $x \in X_{0}$, with $\|x\| \leq 1$,

$$
\left|f\left(T^{-m} x\right)\right|=\left|\tilde{f}\left(T^{-m} x\right)\right| \leq\|\tilde{f}\|_{a_{n}, \mathcal{F}} \cdot\left\|T^{-m} x\right\|_{\mathcal{F}, a_{n}} \leq\|\tilde{f}\|_{\mathcal{F}, a_{n}} \cdot \frac{r^{-m}}{k} .
$$

Thus, $f T^{-m}$ is uniformly bounded on the $\|\cdot\|$-unit ball of $X_{0}$.

Let $g_{m}$ be the unique extension of $f T^{-m}$ to a bounded linear functional on $(X,\|\cdot\|)$. It is easy to verify that $T^{* m} g_{m}=f$ for all $m$. That is, $T^{*^{-m}} f$ exists for all $m$.

e. We have

$$
\begin{aligned}
\left\|T^{*^{-m}} f\right\| \cdot r^{m} & =\sup _{\|x\| \leq 1, x \in X_{0}}\left\|f\left(T^{-m} x\right)\right\| \cdot r^{m} \\
& \leq\|\hat{f}\|_{\mathcal{F}, a_{n}} \cdot\left\|T^{-m} x\right\|_{\mathcal{F}, a_{n}} \cdot r^{m} \leq\|\hat{f}\|_{\mathcal{F}, a_{n}} \cdot \frac{r^{-m}}{k} \cdot r^{m} \leq\|\hat{f}\|_{\mathcal{F}, a_{n}} \cdot \frac{1}{k} .
\end{aligned}
$$

So, $|f| \leq \frac{1}{k} \cdot\|\hat{f}\|$.

f. Let $c>0$ and $C<\infty$ be such that $c \cdot\left\|T^{n}\right\| \leq r^{n} \leq C \cdot\left\|T^{n}\right\| \forall n$. In view of (e) we need only to verify that if $f \in F$, then $f$ has a unique extension $\tilde{f}$ and $\|\tilde{f}\| \leq($ constant $) \cdot|f|$. Let $x \in X$ be such that $\|x\|_{\mathcal{F}, a_{n}} \leq 1$.

$$
\begin{aligned}
|f(x)| & =\left|f\left(T^{-n} T^{n} x\right)\right|=\left|T^{*^{-n}}(f)\left(T^{n} x\right)\right| \leq\left(\left\|T^{*^{-n}} f\right\| \cdot r^{n}\right) \cdot\left(\frac{\left\|T^{n} x\right\|}{r^{n}}\right) \\
& \leq\left(\left\|T^{*^{-n}} f\right\| \cdot r^{n}\right) \cdot\left(\frac{\left\|T^{n} x\right\|}{a_{n}} \cdot \frac{K}{c}\right) .
\end{aligned}
$$

So, $|f(x)| \leq|f| \cdot\|x\|_{a_{n}} \cdot \frac{K}{c}$. Since $X_{0}$ is dense in $\tilde{X}$, it follows that $\|\tilde{f}\|_{\mathcal{F}, a_{n}} \leq \frac{K}{c} \cdot|f|$.

(1) For any $x$ in $X$ we have $(\tilde{T})^{*} \tilde{f}(x)=\tilde{f}(\tilde{T} x)=\tilde{f}(T x)=f(T x)=T^{*} f(x)$. That is, $\left.(\tilde{T})^{*} \tilde{f}\right|_{X}=T^{*} f$ or $(\tilde{T})^{*} \tilde{f}=\left(T^{*} f \tilde{)}\right.$.

(2) Suppose $A$ is invariant under $T^{*}$. Let $\tilde{f} \in J A$. Then $\left.\tilde{f}\right|_{X}=f \in A$. So, $T^{*} f \in A$, and $J\left(T^{*} f\right) \in J A$. By (1) above, $J\left(T^{*} f\right)=(\tilde{T})^{*} \tilde{f}$. So, $(\tilde{T})^{*} \tilde{f} \in J A$. Now suppose $J A$ is invariant under $(\tilde{T})^{*}$. Let $f \in A$. Then $\tilde{f} \in J A$ and $(\tilde{T})^{*} \tilde{f} \in J A$. That is, $J\left(T^{*} f\right) \in J A$. So, $T^{*} f \in A$.

Theorem 8. Suppose $T: X \rightarrow X$ is a bounded linear operator such that $\frac{T}{r}$ is power bounded. Let $F=\left\{x \in(X,\|\cdot\|)^{\prime}: T^{*^{-n}} x\right.$ exists $\forall n$, and $\left.\sup _{n}\left\|T^{*^{-n}} x\right\| r^{n}<\infty\right\}$. Let $a_{n}$ be an admissible sequence for $T$ and $\mathcal{F}$ be any Banach limit invariant under 
the shift. Let $\tilde{T}$ denote the unique extension of $T$ to an operator from the completion $\left(\tilde{X},\|\cdot\|_{\mathcal{F}, a_{n}}\right)$ of $\left(X,\|\cdot\|_{\mathcal{F}, a_{n}}\right)$ to itself. Then, the following hold true.

a. Some nonzero element of $X$ is noncyclic for $\tilde{T}$ iff some nonzero element of $F$ is noncyclic for $T^{*}$.

b. Some nonzero element of $X$ is noncyclic for $(\tilde{T})^{-1}$ iff some nonzero element of $F$ is noncyclic for $\left(T^{*}\right)^{-1}$. (Here, $T$ is assumed to be invertible.)

Proof. a. Suppose some nonzero element of $F$ is noncyclic for $T^{*}$. Then there is a proper closed subspace $M$ of $X^{\prime}$ invariant under $T^{*}$ such that $M \cap F \neq(0)$. Clearly, $M \cap F$ is invariant under $T^{*}$. Let $J$ be an isomorphism as in Lemma 4 (f). Then, $J(M \cap F)$ is invariant under $(\tilde{T})^{*}$ by Lemma 4 (f) (2). Consequently, the polar $[J(M \cap F)]_{\perp \tilde{X}}$ of $J(M \cap F)$ in $\tilde{X}$ is invariant under $\tilde{T}$. We make the following assertions.

(1) $[J(M \cap F)]_{\perp \tilde{X}} \neq \tilde{X}$.

(2) $[J(M \cap F)]_{\perp \tilde{X}} \cap X \neq(0)$.

Clearly, (2) implies that a nonzero element of $X$ is noncyclic for $\tilde{T}$.

Proof of (1). If $[J(M \cap F)]_{\perp \tilde{X}}=\tilde{X}$, then $J(M \cap F)=(0)$. So, $M \cap F=(0)$. This is a contradiction.

Proof of (2). If $[J(M \cap F)]_{\perp \tilde{X}} \cap X=(0)$, then for each $x$ in $X$, there exists an $\tilde{f}$ in $J(M \cap F)$ such that $\tilde{f}(x) \neq 0$. That is, for each $x$ in $X$ there exists $f$ in $M \cap F$ such that $f(x) \neq 0$. Therefore, the closure of $M \cap F$ is $X^{\prime}$. This is a contradiction.

For the converse suppose that some nonzero element of $X$ is noncyclic for $\tilde{T}$. Then there is a proper $\|\cdot\|_{\mathcal{F}, a_{n}}$-closed subspace $M$ of $\tilde{X}$ such that $M \cap X \neq(0)$. Clearly, $M^{\perp} \subset J F$, and $M^{\perp}$ is invariant under $(\tilde{T})^{*}$. By Lemma 4 (f) $(2), J^{-1}\left(M^{\perp}\right)$ is invariant under $T^{*}$. So, the $\|\cdot\|$-closure of $J^{-1}\left(M^{\perp}\right)$ is invariant under $T^{*}$. We claim that $\overline{J^{-1}\left(M^{\perp}\right)}\|\cdot\| \neq X^{\prime}$. Taking the claim for granted, $\overline{J^{-1}\left(M^{\perp}\right)}\|\cdot\|$ is a proper closed subspace invariant under $T^{*}$ and intersecting with $F$. We now prove the claim. If ${\overline{J^{-1}\left(M^{\perp}\right)}}^{\|\cdot\|}=X^{\prime}$, then $\left[J^{-1}\left(M^{\perp}\right)\right]_{\perp X}=(0)$. But $(0) \neq M \cap X \subset$ $\left[J^{-1}\left(M^{\perp}\right)\right]_{\perp X}$.

b can be proved the same way.

\section{NORM CONVERGENCE OF EXTREMAL VECTORS}

Proposition 2 (Duality between the forward and the backward minimal points). Let $T: H \rightarrow H$ be a bounded linear operator.

(a) If $y_{n, x_{0}}^{\epsilon, *}$ is the backward minimal point for $T^{* n}$, and $\epsilon^{\prime}=\left\|T^{* n} y_{n, x_{0}}^{\epsilon, *}\right\|$, then $v_{n, x_{0}}^{\epsilon^{\prime}}=x_{0}-T^{* n} y_{n, x_{0}}^{\epsilon}$ is the forward minimal point for $T^{n}$. Conversely, if $v_{n, x_{0}}^{\epsilon}$ is the forward minimal point for $T^{n}$, and $\epsilon^{\prime}=\left\|v_{n, x_{0}}^{\epsilon}\right\|$, then $T^{* n} y_{n, x_{0}}^{\epsilon^{\prime}}=$ $x_{0}-v_{n, x_{0}}^{\epsilon}$ is the backward minimal point for $T^{* n}$.

(b) Let $x_{0}$ be a fixed vector in $H$ with $\left\|x_{0}\right\|=1$. Let $\beta_{n}^{\epsilon}$ be the angle between $T^{n} y_{n, x_{0}}^{\epsilon}$ and $T^{n} y_{n, x_{0}}^{\epsilon}-x_{0}, \alpha_{n}^{*, \epsilon}$ be the angle between $v_{n, x_{0}}^{*, \epsilon}$ and $\left(v_{n, x_{0}}^{*, \epsilon}-x_{0}\right)$, and $\alpha_{n}^{\epsilon}$ be the angle between $v_{n, x_{0}}^{\epsilon}$ and $\left(v_{n, x_{0}}^{\epsilon}-x_{0}\right)$. Then,

(1) $\lim _{\epsilon \rightarrow 1} \operatorname{Cos} \beta_{n}^{\epsilon}=\lim _{\epsilon \rightarrow 0} \operatorname{Cos} \alpha_{n}^{*, \epsilon}$, and

(2) $\lim _{\epsilon \rightarrow 0} \operatorname{Cos} \alpha_{n}^{\epsilon} \leq \frac{-\left\|T^{n} x_{0}\right\|}{\left\|T^{n}\right\|}$.

(c) Suppose for each $0<\epsilon<\left\|x_{0}\right\|$ and for each sequence $\left(\epsilon_{n_{k}}\right)$ converging to $\epsilon, v_{n_{k}}^{\epsilon_{n_{k}}} \rightarrow v_{x_{0}}$ in norm. Then, there exists a subsequence $\left(n_{k_{l}}\right)$ such that 
$T^{*^{n k_{l}}} y_{n_{k_{l}}, x_{0}}^{*, \epsilon} \rightarrow y_{x_{0}}^{*}$ in norm, where $T^{*^{n k_{l}}} y_{n_{k_{l}}, x_{0}}^{*, \epsilon}$ is the backward minimal vector for $T^{*^{n_{k}}}$.

(d) Let $0<\epsilon<\left\|x_{0}\right\|$. Suppose for each sequence $\left(\epsilon_{n_{k}}\right)$ converging to $\epsilon, T^{*^{n_{k}}} y_{n_{k}, x_{0}}^{*, \epsilon_{n_{k}}}$ $\rightarrow y_{x_{0}}^{*}$ in norm. Then, there exists a subsequence $\left(n_{k_{l}}\right)$ such that $v_{n_{k_{l}}}^{\epsilon} \rightarrow v_{x_{0}}$ in norm.

Proof. (a) Let $y_{n, x_{0}}^{\epsilon, *}=y_{n}^{*}$. $y_{n}^{*}$ satisfies the equation $T^{*^{n}} y_{n}^{*}=\delta_{n} T^{*^{n}} T^{n}\left(T^{*^{n}} y_{n}^{*}-x_{0}\right)$ for some $\delta_{n}<0$. Set $v_{n}=x_{0}-T^{*^{n}} y_{n}^{*}$. Then, $-T^{*^{n}} y_{n}^{*}=\delta_{n} T^{*^{n}} T^{n} v_{n}$. So, $\left(v_{n}-x_{0}\right)=\delta_{n} T^{*^{n}} T^{n} v_{n}$. Since the equation $\left(X-x_{0}\right)=a T^{*^{n}} T^{n} X$ has a unique solution of the form $(a, X)$ for $a<0$, it follows that $v_{n}$ is a forward minimal point. As the $\left\|v_{n}-x_{0}\right\|=\left\|T^{*^{n}} y_{n}^{*}\right\|=\epsilon^{\prime}, \quad v_{n}=v_{n, x_{0}}^{\epsilon^{\prime}}$.

(b) Let $y_{n}^{\epsilon}=y_{n, x_{0}}^{\epsilon}, v_{n}^{\epsilon}=v_{n, x_{0}}^{\epsilon}$, and $v_{n}^{*, \epsilon}=v_{n, x_{0}}^{*, \epsilon}$ and $\epsilon^{\prime}=\left\|T^{n} y_{n}^{\epsilon}\right\|$. We have $\left(T^{n} y_{n}^{\epsilon}-x_{0}, T^{n} y_{n}^{\epsilon}\right)=\left(-v_{n}^{*, \epsilon^{\prime}}, x_{0}-v_{n}^{*, \epsilon^{\prime}}\right)$. So, $\epsilon \cdot\left\|T^{n} y_{n}^{\epsilon}\right\| \cdot \operatorname{Cos} \beta_{n}^{\epsilon}==\epsilon^{\prime} \cdot\left\|v_{n, x_{0}}^{*, \epsilon^{\prime}}\right\| \cdot \operatorname{Cos} \alpha_{n}^{*, \epsilon}$. Therefore, $\operatorname{Cos} \beta_{n}^{\epsilon}=\frac{\left\|v_{n}^{*} \epsilon^{\prime}\right\| \cdot \operatorname{Cos} \alpha_{n}^{\epsilon}}{\epsilon}$. As $\epsilon \rightarrow 1, \quad \epsilon^{\prime} \rightarrow 0$, and $\left\|v_{n}^{*, \epsilon}\right\| \varnothing 1$. The first equation is now immediate.

We have $\left(v_{n}^{\epsilon}-x_{0}, v_{n}^{\epsilon}\right)=\epsilon \cdot\left\|v_{n}^{\epsilon}\right\| \cdot \cos \alpha_{n}^{\epsilon}$. On the other hand, from the orthogonality equation

$$
\left(v_{n}^{\epsilon}-x_{0}, v_{n}^{\epsilon}\right)=\left(\delta_{n}^{\epsilon} T^{* n} T^{n} v_{n}^{\epsilon}, v_{n}^{\epsilon}\right)=\delta_{n}^{\epsilon}\left\|T^{n} v_{n}\right\|^{2} .
$$

So,

$\cos \alpha_{n}^{\epsilon}=\frac{\delta_{n}^{\epsilon}\left\|T^{n} v_{n}\right\|^{2}}{\epsilon\left\|v_{n}^{\epsilon}\right\|^{2}}=\frac{-\left\|T^{n} v_{n}\right\|^{2}}{\left\|v_{n}^{\epsilon}\right\|^{2} \cdot\left\|T^{* n} T^{n} v_{n}\right\|} \leq \frac{-\left\|T^{n} v_{n}\right\|^{2}}{\left\|v_{n}\right\|^{2} \cdot\left\|T^{n}\right\| \cdot\left\|T^{n} v_{n}\right\|}=\frac{-\left\|T^{n} v_{n}\right\|}{\left\|v_{n}\right\|^{2} \cdot\left\|T^{n}\right\|}$.

Note that

$$
\lim _{\epsilon \rightarrow 0} \frac{-\left\|T^{n} v_{n}\right\|}{\left\|v_{n}\right\|^{2} \cdot\left\|T^{n}\right\|}=\frac{-\left\|T^{n} x_{0}\right\|}{\left\|T^{n}\right\|} .
$$

(c) Let $\left(n_{k_{l}}\right)$ be a subsequence such that $\left(\left\|T^{*^{n}{ }^{n}{ }_{l}} y_{n_{k_{l}}}^{*, \epsilon}\right\|\right)=\left(\epsilon_{n_{k_{l}}}\right)$ converges in norm to say $\epsilon_{0}$. Then, $0<\epsilon_{0}<\left\|x_{0}\right\|$. Now the result follows from (a).

(d) This is similar to (c).

Thus, if $x_{0}$ is quasimaximal for $T$ and $0<\delta<\frac{\left\|T^{n} x\right\|}{\left\|T^{n}\right\|}$ for all $n$, then for some $\delta^{\prime}>0, \lim _{\epsilon \rightarrow 0} \operatorname{Cos} \alpha_{n, x_{0}}^{\epsilon} \leq-\delta^{\prime} \forall n$. Example 2 (below) shows that $x_{0}$ does not have to be quasimaximal for $\lim _{\epsilon \rightarrow 0} \operatorname{Cos} \alpha_{n, x_{0}}^{\epsilon} \leq-\delta^{\prime}$ to hold true.

Our definition of minimal points make sense if the space is a strictly convex reflexive Banach space. More precisely, if $X$ is reflexive and $T: X \rightarrow X$ is a bounded operator, then the set $\left\{T^{n} y:\left\|y-x_{0}\right\| \leq \epsilon\right\}$ is weakly compact for any $x_{0} \in X$ and $0<\epsilon<\left\|x_{0}\right\|$. So, the norm of $X$ attains its minimum over this set at a point of the set. If the norm of $X$ is strictly convex, then the minimum is attained at a unique element of this set. That unique element can be defined to be the forward minimal point $v_{n, x_{0}}^{\epsilon}$. Similarly, the definition of backward minimal points can be extended.

Recall that a result of M. I. Kadets [2] states that any separable Banach space admits an equivalent new norm which is locally uniformly convex. Recall also that a locally uniformly convex norm is strictly convex and has the property that if $\left(x_{n}\right)$ is a sequence such that $x_{n} \rightarrow x$ weakly and $\left\|x_{n}\right\| \rightarrow\|x\|$, then $x_{n} \rightarrow x$ in norm.

Proposition 3. Suppose $X$ is a reflexive locally uniformly convex Banach space and $T: X \rightarrow X$ is any bounded linear operator. Let $v_{n}=v_{n, x_{0}}^{\epsilon}$. 
a. If $v_{n_{k}} \rightarrow v_{0}$ weakly and if $\frac{\left\|T^{n} v_{n_{k}}\right\|}{\left\|T^{n} k v_{0}\right\|} \rightarrow 1$, then $v_{n_{k}} \rightarrow v_{0}$ in norm.

b. Suppose $T$ is of class $\mathcal{R},\left(a_{n}\right)$ is an admissible sequence for $T$, and $\mathcal{F}$ is any Banach limit. If $v_{n_{k}} \rightarrow v_{0}$ in norm, then

1. $\frac{\left\|T^{n} k v_{n_{k}}\right\|}{\left\|T^{n} k v_{0}\right\|} \rightarrow 1$ and

2. $\left\|v_{0}\right\|_{\mathcal{F}, a_{n_{k}}} \leq\|x\|_{\mathcal{F}, a_{n_{k}}}$.

Proof. (a) Suppose not. Since $X$ is locally uniformly convex, $\left\|v_{0}-x_{0}\right\|<\epsilon$. So, $\left\|t v_{0}-x_{0}\right\|=\epsilon$ for some $0<t<1$. Consequently, $\frac{\left\|T^{n_{k}} v_{n_{k}}\right\|}{t\left\|T^{n} v_{0}\right\|} \rightarrow \frac{1}{t}>\delta>1$ for some $\delta$. That is, $\left\|T^{n_{k}} v_{n_{k}}\right\|>\delta\left\|T^{n_{k}}\left(t v_{0}\right)\right\| \quad \forall k$ large enough. This contradicts the definition of $v_{n_{k}}$.

(b) (1) We have

$$
\frac{\left\|T^{n_{k}} v_{0}\right\|}{\left\|T^{n_{k}}\right\|}-\frac{\left\|T^{n_{k}} v_{n_{k}}\right\|}{\left\|T^{n_{k}}\right\|} \leq\left\|\frac{T^{n_{k}} v_{0}}{\left\|T^{n_{k}}\right\|}-\frac{T^{n_{k}} v_{n_{k}}}{\left\|T^{n_{k}}\right\|}\right\| \leq\left\|v_{n_{k}}-v_{0}\right\| \rightarrow 0 .
$$

So,

$$
\left(1-\frac{\left\|T^{n_{k}} v_{n_{k}}\right\|}{\left\|T^{n_{k}} v_{0}\right\|}\right) \frac{\left\|T^{n_{k}} v_{0}\right\|}{\left\|T^{n_{k}}\right\|} \rightarrow 0 .
$$

Since, $\frac{\left\|T^{n_{k}} v_{0}\right\|}{\left\|T^{n} k\right\|} \geq \delta>0$, it follows that $\frac{\left\|T^{n} v_{n_{k}}\right\|}{\left\|T^{n} v_{0}\right\|} \rightarrow 1$.

(2) Let $\frac{\left\|T^{n_{k}} v_{n_{k}}\right\|}{\left\|T^{n} v_{0}\right\|}=\delta_{n_{k}}$. Then, $\delta_{n_{k}}<1 \forall k$ and $\delta_{n_{k}} \rightarrow 1$. For any $x \in \overline{B\left(x_{0}, \epsilon\right)}$ we have $\left\|T^{n_{k}} x\right\| \geq\left\|T^{n_{k}} v_{n_{k}}\right\|=\delta_{n_{k}}\left\|T^{n_{k}} v_{0}\right\|$. So,

$$
\frac{\left\|T^{n_{k}} x\right\|}{a_{n_{k}}} \geq \frac{\left\|T^{n_{k}} v_{n_{k}}\right\|}{a_{n_{k}}}=\delta_{n_{k}} \frac{\left\|T^{n_{k}} v_{0}\right\|}{a_{n_{k}}} .
$$

It follows that $\|x\|_{\mathcal{F}, a_{n_{k}}} \geq\left\|v_{0}\right\|_{\mathcal{F}, a_{n_{k}}}$.

A corollary of Theorem 11, which appears in the appendix, is given bellow.

Corollary. Suppose $X$ is a strictly convex smooth reflexive Banach space, $T: X \rightarrow$ $X$ is of class $\mathcal{R},\left(a_{n}\right)$ and $\left(b_{n}\right)$ are any admissible sequences and $\mathcal{F}$ is any Banach limit. If for each $x_{0} \neq 0$ and each $0<\epsilon<\left\|x_{0}\right\|$, the sequence $v_{n, x_{0}}^{\epsilon} \rightarrow v_{x_{0}}^{\epsilon}$ in norm, then there exists a $K$ such that $\|\cdot\|_{\mathcal{F}, a_{n}}=K \cdot\|\cdot\|_{\mathcal{F}, b_{n}}$.

Theorem 9. Suppose $(X,\|\cdot\|)$ is a reflexive locally uniformly convex Banach space, $T: X \rightarrow X$ is an operator of class $\mathcal{R}$ and $\left(a_{n}\right)$ is an admissible sequence for $T$. Let $x_{0} \in X, 0<\epsilon, \epsilon_{n}<\left\|x_{0}\right\|, \epsilon_{n} \rightarrow \epsilon$, and $v_{n}^{\epsilon_{n}}=v_{n, x_{0}}^{\epsilon_{n}}$.

a. Suppose for a weak limit point $v_{0}$ of $\left\{v_{n}^{\epsilon_{n}}: n \geq 1\right\}$ there is a norm $\|\cdot\|_{v_{0}}$ on $X$ such that $\left\|v_{0}\right\|_{v_{0}} \leq\|x\|_{v_{0}} \forall x \in \overline{B\left(x_{0}, \epsilon\right)_{\|\cdot\|}}$. Then $v_{0}$ is a norm limit point of $\left\{v_{n}^{\epsilon_{n}}: n \geq 1\right\}$.

b. Suppose for each $k$ the sequence $\left(\frac{\left\|T^{n} v_{k}^{\epsilon_{k}}\right\|}{a_{n}}\right)_{n=1}^{k}$ is increasing. Then, for each weak limit point $v_{0}$ of $\left\{v_{n}^{\epsilon_{n}}: n \geq 1\right\}$, there is a norm $\|\cdot\|_{v_{0}}$ on $X$ such that $\left\|v_{0}\right\|_{v_{0}} \leq\|x\|_{v_{0}} \forall x \in \overline{B\left(x_{0}, \epsilon\right)_{\|\cdot\|}}$. Consequently, each weak limit point of $\left\{v_{n}^{\epsilon_{n}}: n \geq 1\right\}$ is a norm limit point of it.

c. Suppose $\lim _{n} \frac{\left\|T^{n} v_{k}^{\epsilon_{k}}\right\|}{a_{n}}$ exists and $\lim _{n} \frac{\left\|T^{n} v_{k}^{\epsilon_{k}}\right\|}{a_{n}} \leq \frac{\left\|T^{k} v_{k}^{\epsilon_{k}}\right\|}{a_{k}}$ for each $k$. Then, for each weak limit point $v_{0}$ of $\left\{v_{n}^{\epsilon_{k}}: n \geq 1\right\}$, there is a norm $\|\cdot\|_{v_{0}}$ on $X$ such that $\left\|v_{0}\right\|_{v_{0}} \leq\|x\|_{v_{0}} \forall x \in \overline{B\left(x_{0}, \epsilon\right)_{\|\cdot\|}}$. Consequently, each weak limit point of $\left\{v_{n}^{\epsilon_{n}}: n \geq 1\right\}$ is a norm limit point of it. 
d. Suppose $\lim _{n} \frac{\left\|T^{n} x\right\|}{a_{n}}$ exists for all $x \in X$ and $\lim _{n} \frac{\left\|T^{n} v_{k}^{\epsilon_{k}}\right\|}{a_{n}} \leq \frac{\left\|T^{k} v_{k}^{\epsilon_{k}}\right\|}{a_{k}}$ for each $k$. Then, $v_{n}^{\epsilon_{n}} \rightarrow v_{0}$ in norm for some $v_{0}$. In particular, if $T$ is a $C_{1}$ contraction, then $\left(v_{n, x_{0}}^{\epsilon}\right)$ converges in norm for each $x_{0} \neq 0$ and $0<\epsilon<\left\|x_{0}\right\|$.

Proof. (a) Suppose not. Since, $(X,\|\cdot\|)$ is locally uniformly convex, it follows that $\left\|v_{0}-x_{0}\right\|<\epsilon$. So, for some $t<1,\left\|t v_{0}-x_{0}\right\|=\epsilon$. By the hypothesis, it follows that $\left\|v_{0}\right\|_{v_{0}} \leq t\left\|v_{0}\right\|_{v_{0}}$. This is a contradiction.

(b) Let $\mathcal{F}$ be any Banach limit. Let $v_{0}$ be any weak limit point of the set and let $v_{n_{k}}^{\epsilon_{n_{k}}} \rightarrow v_{0}$ weakly. For any $m$ we have

$$
\begin{aligned}
\frac{\left\|T^{m} v_{0}\right\|}{a_{m}} & \leq \lim \inf _{k} \frac{\left\|T^{m} v_{n_{k}}^{\epsilon_{n_{k}}}\right\|}{a_{m}} \\
& \leq \liminf _{k} \frac{\| T^{n_{k}} v_{n_{k}} \epsilon_{n_{k}}}{a_{n_{k}}} \\
& \left.=\lim _{l} \frac{\| T^{n_{k_{l}}} v_{n_{n_{l}}} \epsilon_{n_{l}}}{a_{n_{k_{l}}}} \quad \text { (for some subsequence }\left(n_{k_{l}}\right) \text { of }\left(n_{k}\right)\right) \\
& \leq \lim \inf _{l} \frac{\left\|T^{n_{k_{l}}} x\right\|}{a_{n_{k_{l}}}} \quad \text { for each } x \in B\left(x_{0}, \epsilon\right)
\end{aligned}
$$

Define $\|x\|_{v_{0}}=\mathcal{F}\left(\frac{\left\|T^{n_{k}} x\right\|}{a_{n_{k_{l}}}}\right)$ for all $x \in X$. It follows that

$$
\mathcal{F}\left(\frac{\left\|T^{n_{k_{l}}} v_{0}\right\|}{a_{n_{k_{l}}}}\right) \leq \mathcal{F}\left(\frac{\| T^{n_{k_{l}} x \|}}{a_{n_{k_{l}}}}\right) .
$$

That is, $\left\|v_{0}\right\|_{v_{0}} \leq\|x\|_{v_{0}} \quad \forall x \in B\left(x_{0}, \epsilon\right)$. Since $\|\cdot\|_{v_{0}} \leq($ const $)\|\cdot\|$, it follows that $\left\|v_{0}\right\|_{v_{0}} \leq\|x\|_{v_{0}} \quad \forall x \in \overline{B\left(x_{0}, \epsilon\right)_{\|\cdot\|}}$.

(c) Let $v_{0}$ be any weak limit of the set and let $v_{n_{k_{l}}}^{\epsilon_{n_{k_{l}}}} \rightarrow v_{0}$ weakly. Define $\|x\|_{v_{0}}=\mathcal{F}\left(\frac{\| T^{n_{k} x \|}}{a_{n_{k}}}\right)$. Since $\|x\|_{v_{0}} \leq$ (constant) $\cdot\|x\|$ for all $x \in X$, it follows that $v_{n_{k}}^{\epsilon_{n_{k}}} \rightarrow v_{0}$ weakly in $\left(X,\|\cdot\|_{v_{0}}\right)$. Thus,

$$
\begin{aligned}
\left\|v_{0}\right\|_{v_{0}} & \leq \lim \inf _{k}\left\|v_{n_{k}}^{\epsilon_{n_{k}}}\right\|_{v_{0}} \leq \lim \inf _{k} \frac{\left\|T^{n_{k}} v_{n_{k}}^{\epsilon_{n_{k}}}\right\|}{a_{n_{k}}} \\
& \leq \lim \inf _{k} \frac{\left\|T^{n_{k}} x\right\|}{a_{n_{k}}} \quad \forall x \in \overline{B\left(x_{0}, \epsilon\right)_{\|\cdot\|}} .
\end{aligned}
$$

It follows that $\left\|v_{0}\right\|_{v_{0}} \leq\|x\|_{v_{0}}$ for all $x \in \overline{B\left(x_{0}, \epsilon\right)_{\|\cdot\|}}$.

(d) Let $v_{0}$ be any weak limit of the sequence $\left(v_{n}\right)$. Define $\|x\|_{a_{n}}=\lim _{n} \frac{\left\|T^{n} x\right\|}{a_{n}}$ $\forall x \in X$. Let $v_{n_{k}}^{\epsilon_{n_{k}}} \rightarrow v_{0}$ weakly in $(X,\|\cdot\|)$. Then,

$$
\begin{aligned}
\left\|v_{0}\right\|_{a_{n}} & \leq \lim \inf _{k}\left\|v_{n_{k}}^{\epsilon_{n_{k}}}\right\|_{a_{n}}=\lim \inf _{k} \lim _{m} \frac{\left\|T^{m} v_{n_{k}}^{\epsilon_{n_{k}}}\right\|}{a_{m}} \\
& \leq \lim \inf _{k} \frac{\left\|T^{n_{k}} v_{n_{k}}^{\epsilon_{n_{k}}}\right\|}{a_{n_{k}}} \leq \lim \inf _{k} \frac{\left\|T^{n_{k}} x\right\|}{a_{n_{k}}} .
\end{aligned}
$$

It follows that $\left\|v_{0}\right\|_{a_{n}} \leq\|x\|_{a_{n}}$. By (a) it follows that $v_{n_{k}}^{\epsilon_{n_{k}}} \rightarrow v_{0}$ in norm. Now suppose if possible that $z_{0}$ is a norm limit point of the set $\left\{v_{n}: n \geq 1\right\}$ and $v_{0} \neq z_{0}$. By strict convexity of $\|\cdot\|,\left\|\frac{v_{0}+z_{0}}{2}-x_{0}\right\|<\epsilon$. So, for some $0<t<1$ we have 
$t\left(\frac{v_{0}+z_{0}}{2}\right) \in \overline{B\left(x_{0}, \epsilon\right)_{\|\cdot\|}}$. Note that $\left\|v_{0}\right\|_{a_{n}}=\left\|z_{0}\right\|_{a_{n}}$. So, $t\left\|v_{0}\right\|_{a_{n}} \geq\left\|t\left(\frac{v_{0}+z_{0}}{2}\right)\right\|_{a_{n}} \geq$ $\left\|v_{0}\right\|_{a_{n}}$. This is a contradiction.

Theorem 10. Let $X$ be a reflexive locally uniformly convex Banach space. Let $T: X \rightarrow X$ be such that $\|T\|=1$.

a. Suppose there are $x_{0} \in X$ and $0<\epsilon<\left\|x_{0}\right\|$ such that $\left(y_{n, x_{0}}^{\epsilon}\right)$ is bounded. Then, $y_{0} \in F=\left\{x: T^{-n} x\right.$ exists $\left.\forall n, \operatorname{Sup}_{n}\left\|T^{-n} x\right\|<\infty\right\}$ for each weak limit $y_{0}$ of $\left(T^{n} y_{n, x_{0}}^{\epsilon}\right)$.

b. Suppose the vector space $F=\left\{x: T^{-n} x\right.$ exist $\forall n$, Sup $\left.n\left\|T^{-n} x\right\|<\infty\right\}$ is dense. Let $x_{0} \in X$ and $\epsilon>0$ be such that $0<\epsilon<\left\|x_{0}\right\|$. Then the following hold.

1. $\left(y_{n, x_{0}}^{\epsilon}\right)$ is a bounded sequence.

2. $T^{n} y_{n, x_{0}}^{\epsilon} \rightarrow y_{0}$ in norm for some $y_{0}$.

3. $y_{0} \in F=\left\{x: T^{-n} x\right.$ exist $\forall n$, Sup $\left._{n}\left\|T^{-n} x\right\|<\infty\right\}$.

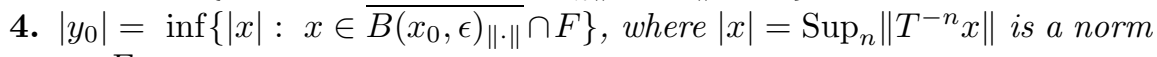
on $F$.

Proof. (a). Let $y_{n}=y_{n, x_{0}}^{\epsilon}$ for all $n$. Suppose $\left\|y_{n}\right\| \leq M \forall n$. Then, $\left\|T^{k} y_{n}\right\| \leq M$ $\forall n, k \geq 1$. Suppose $z_{0}$ is a weak limit point of $\left(T^{n} y_{n}\right)$. Since $\left\|z_{0}-x_{0}\right\|=\epsilon<$ $\left\|x_{0}\right\|, z_{0} \neq 0$. We can find a subsequence $\left(n_{k}\right)$ of natural numbers such that $T^{n_{k}-m} y_{n_{k}} \rightarrow z_{m}$ weakly for some $z_{m}$ for all $m \geq 0$. Clearly, $z_{m}=T^{-m} z_{0}$ and $\left\|T^{-m} z_{0}\right\|=\left\|z_{m}\right\| \leq \sup \left\|y_{n}\right\| \leq M$. This implies $z_{0} \in F$.

(b) Let $y_{n}=y_{n, x_{0}}^{\epsilon}$ for all $n$. Since the space $F$ is dense, the sequence $\left(y_{n}\right)$ is bounded. Suppose $T^{n_{k}} y_{n_{k}} \rightarrow y_{0}$ for some $y_{0}$ weakly. By (a), $y_{0} \in F$.

Claim. $\left|y_{0}\right|=\inf \left\{|x|: x \in \overline{B\left(x_{0}, \epsilon\right)_{\|\cdot\|}} \cap F\right\}$.

Proof of the claim. Let $\left(n_{l}\right)$ be a subsequence of $\left(n_{k}\right)$ such that $T^{n_{l}-m} y_{n_{l}} \rightarrow T^{-m} y_{0}$ for each $m \geq 1$. We have

$$
\begin{aligned}
\left\|T^{-m} y_{0}\right\| & \leq \lim \inf _{l}\left\|T^{n_{l}-m} y_{n_{l}}\right\| \\
& \leq \lim \inf _{l}\left\|y_{n_{l}}\right\| \\
& \leq \lim \inf _{l}\left\|T^{-n_{l}} x\right\| \text { for each } x \in \overline{B\left(x_{0}, \epsilon\right)_{\|\cdot\|}} \cap F \\
& \leq \sup _{n}\left\|T^{-n} x\right\| \text { for each } x \in \overline{B\left(x_{0}, \epsilon\right)_{\|\cdot\|}} \cap F
\end{aligned}
$$

Therefore, $\left|y_{0}\right| \leq|x|$ for each $x \in \overline{B\left(x_{0}, \epsilon\right)_{\|\cdot\|}} \cap F$.

Now an argument similar to the one in the proof of Theorem 9 (a) implies that $T^{n_{k}} y_{n_{k}} \rightarrow y_{0}$ in norm. Further, as in the proof of Theorem 9 (d), the strict convexity of $\|\cdot\|$ implies that $T^{n} y_{n} \rightarrow y_{0}$ in norm.

The next remark follows from Theorem 10 (b).

Remark 7. Let $X$ be a Banach space, let $X^{\prime}$ have a locally uniformly convex norm, and let $T: X \rightarrow X$ be a $C_{1}$-contraction. Then, by Lemma 4 (a) $F=\left\{x \in X^{\prime}\right.$ : $T^{*^{-n}} x$ exists $\left.\forall n, \operatorname{Sup}_{n}\left\|T^{*^{-n}} x\right\|<\infty\right\} \neq\{0\}$. If $F$ is not dense in $(X,\|\cdot\|)^{\prime}$, then $\bar{F}$ is an invariant subspace for $T$. Suppose $F$ is dense in $(X,\|\cdot\|)^{\prime}$. Let $x_{0} \in X^{\prime}$ and $0<\epsilon<\left\|x_{0}\right\|$. Let $T^{* n} y_{n}^{*}$ denote the backward minimal points w.r.t. $\left(x_{0}, \epsilon, T^{* n}\right)$. Then, the following hold.

a. $T^{* n} y_{n}^{*} \rightarrow y_{0}$ in norm for some $y_{0}$. 
b. $y_{0} \in F=\left\{x \in X^{\prime}: T^{*^{-n}} x\right.$ exists $\left.\forall n, \sup _{n}\left\|T^{*^{-n}} x\right\|<\infty\right\}$.

Remark 8. Suppose $T: H \rightarrow H$ is a $C_{1}$-contraction. If $H$ does not contain any nonzero noncyclic vector for $\tilde{T}$, then by Theorem 8 and Remark 6 it follows that all the norm limits of the sequences $\left(T^{*^{n}} y_{n, x_{0}}^{\epsilon, *}\right)$ are cyclic vectors for $T^{*}$. B. Beauzamy defined an invertible $C_{1}$-contraction $M_{\phi}$ on Hilbert space $H$ and proved that no nonzero vector of $H$ is noncyclic for $\tilde{M}_{\phi}$, where $\tilde{M}_{\phi}$ is the unique extension of $M_{\phi}$ to an operator on $(\tilde{H},\|\| \cdot\|\|)$ and $\left\|\left|x\left\|\mid=\lim _{n \rightarrow \infty}\right\| T^{n} x \|\right.\right.$ (see [1]). (Note that $\||| \cdot||=\| \cdot \|_{\mathcal{F},\left\|T^{n}\right\|}$ for any Banach limit $\mathcal{F}$.) Thus, the norm limit of $\left(M_{\phi}^{* n} y_{n, x_{0}}^{\epsilon}\right)$ is cyclic for $M_{\phi}^{*}$ for each $x_{0} \neq 0$ and $0<\epsilon<\left\|x_{0}\right\|$. It is worth mentioning that by a result of B. Beauzamy [1, Ch. XII], for $T$ belonging to a large class of $C_{1}$-contractions on a Banach space $X$ (not necessarily locally uniformly convex), the space $X$ contains a nonzero noncyclic vector for $\tilde{T}$. So, by Theorem 8 for $T$ belonging to a large class of $C_{1}$-contractions on $X$, the space $F=\{x \in X$ : $T^{*^{-n}} x$ exists $\left.\forall n, \operatorname{Sup}_{n}\left\|T^{*^{-n}} x\right\|<\infty\right\}$ contains a nonzero noncyclic vector of $T^{*}$.

Example 1. We define an operator $T: l^{2} \rightarrow l^{2}$ such that $T$ is of class $\mathcal{R},\left\|T^{n}\right\| \rightarrow$ $\infty$ and for any real number $t \neq 1$, either $\left\|\left(\frac{T}{t}\right)^{n}\right\| \rightarrow 0$ or $\left\|\left(\frac{T}{t}\right)^{n}\right\| \rightarrow \infty$. Let $\left\{e_{n}: n=0,1,2, \ldots\right\}$ be an orthonormal basis for $l^{2}$. Let $\left(a_{n}\right)$ be a sequence of positive real numbers such that $a_{0} \cdot a_{1} \cdot a_{2} \cdots a_{n}=(n+1)^{k}$. Define $T e_{n}=a_{n} \cdot e_{n+1}$ and extend $T$ to all of $l^{2}$ to be a bounded linear operator. It is easy to check that $T$ is of class $\mathcal{R},\left\|T^{n}\right\|=n^{k}, r(T)=1$, for $0<t<1,\left\|\left(\frac{T}{t}\right)^{n}\right\| \rightarrow \infty$, and for $t>1$, $\left\|\left(\frac{T}{t}\right)^{n}\right\| \rightarrow 0$

Example 2. Let $T$ be a bilateral weighted shift defined by $T e_{n}=a_{n} e_{n+1}$ for all integers $n$. Let $x_{0}=\sum_{i=-k}^{k} c_{i} e_{i}$ and $0<\epsilon<\left\|x_{0}\right\|$. Then every subsequence of $\left(T^{n} y_{n}\right)$ has a norm convergent subsequence. Every subsequence of $\left(v_{n}\right)$ has a norm convergent subsequence. If $x_{0}=e_{k}$ for any $k$, then $T^{n} y_{n}=(1-\epsilon) e_{k}$ and $v_{n}=(1-\epsilon) e_{k}$ for all $n$.

Proof. Note that $T^{* n} T^{n} e_{i}=\left|a_{i}\right|^{2} \cdots\left|a_{i+n-1}\right|^{2} \cdot e_{i} \forall n$ and $\forall i$. Let $v_{n}=\sum_{-\infty}^{\infty} b_{i} e_{i}$. We have $\left(v_{n}-x_{0}\right)=\delta_{n} T^{* n} T^{n} v_{n}$, where $\delta_{n}<0$. We get

$$
\sum_{-\infty}^{\infty} b_{i} e_{i}-\sum_{-k}^{k} c_{i} e_{i}=\delta_{n} \sum_{-\infty}^{\infty}\left|a_{i}\right|^{2} \cdots\left|a_{i+n-1}\right|^{2} b_{i} e_{i} .
$$

So, $b_{i}=\delta_{n}\left|a_{i}\right|^{2} \cdots\left|a_{i+n-1}\right|^{2} b_{i}$ for $i<-k$ and $i>k$. Consequently, $\delta_{n}<0$ implies that $b_{i}=0$ for $i<-k$ and $i>k$. This means that the $v_{n}$ are all contained in a finite dimensional space. The conclusion regarding $\left(v_{n}\right)$ is now clear. A similar argument works for $\left(T^{n} y_{n}\right)$. The assertion regarding the case $x_{0}=e_{k}$ is also now clear. Clearly, the angle between $v_{n, e_{k}}^{\epsilon}$ and $\left(v_{n, e_{k}}^{\epsilon}-x_{0}\right)$ is $\pi$, and the same holds for the angle between $T^{n} y_{n, e_{k}}^{\epsilon}$ and $\left(T^{n} y_{n, e_{k}}^{\epsilon}-x_{0}\right)$. So, in the terminology of Proposition $2, \operatorname{Cos} \alpha_{n, e_{k}}^{\epsilon}=\operatorname{Cos} \beta_{n, e_{k}}^{\epsilon}=-1 \forall n$ and for all $0<\epsilon<1$.

Example 3. In this example $v_{n, x_{0}}^{\epsilon}=u$ for all odd integers $n$, and $v_{n, x_{0}}^{\epsilon}=w$ for all even integers $n$ and $u \neq w$. That is, in this case the sequence $\left(v_{n}\right)$ splits into two subsequences, one converging in norm to one point and the other to another point. Let $\left(a_{n}\right)$ be a sequence such that $a_{2 n}=2 \forall n \geq 0$ and $a_{2 n+1}=\frac{1}{2} \forall n \geq 0$. Let $\left\{e_{n}: n \geq 0\right\}$ be an orthonormal besis for $l^{2}$. Define $T$ on $l^{2}$ by $T e_{n}=a_{n} e_{n+1}$. Let $x_{0}=e_{1}+e_{2}$ and $\epsilon=1$. From Example 2 it is clear that $v_{n}=b_{n} e_{1}+c_{n} e_{2}$ for some constants $a_{n}$ and $b_{n} \forall n$. Easy computations show that 
$T^{* n} T^{n} v_{n}=\frac{b_{n}}{4} e_{1}+4 c_{n} e_{2}$ if $n$ is odd and

$T^{* n} T^{n} v_{n}=b_{n} e_{1}+c_{n} e_{2}$ if $n$ is even.

From the orthogonality equation we get

$\left(b_{n}-1\right) e_{1}+\left(c_{n}-1\right) e_{2}=\delta_{n}\left(\frac{b_{n}}{4} e_{1}+4 c_{n} e_{2}\right)$ if $n$ is odd and

$\left(b_{n}-1\right) e_{1}+\left(c_{n}-1\right) e_{2}=\delta_{n}\left(b_{n} e_{1}+c_{n} e_{2}\right)$ if $n$ is even.

Thus $\left(b_{n}, c_{n}\right)$ satisfy the equations

(1) $(X-1)^{2}+(Y-1)^{2}=1$ and

(2) $1-\frac{1}{X}=\frac{1}{16}-\frac{1}{16 Y}$ if $n$ is odd

and the equations

(3) $(X-1)^{2}+(Y-1)^{2}=1$ and

(4) $1-\frac{1}{X}=1-\frac{1}{Y}$ if $n$ is even.

Let $u=\left(u_{1}, u_{2}\right)$ and $w=\left(w_{1}, w_{2}\right)$ be the solutions to the equations (1), (2), and (3), (4), respectively.

An operator $T$ can be defined in a similar way so that for some $x_{0}$ and $\epsilon<\left\|x_{0}\right\|$, the sequence $\left(T^{n} y_{n, x_{0}}^{\epsilon}\right)$ behaves like $\left(v_{n}\right)$ does in the above example.

\section{APPENDIX}

Definition. Let $X$ be a normed linear space. Let $f$ be a continuous linear functional and $x$ be a nonzero vector in $X$. The hyperplane $x+\operatorname{Ker} f$ is said to be norming at $X$ if $\|x+h\| \geq\|x\|$ for all $h \in$ Ker $f$. For any $\delta>0$ the hyperplane $x+$ Ker $f$ is said to be $(1+\delta)$-norming at $x$ if $\|x+h\| \geq \frac{1}{1+\delta} \cdot\|x\|$ for all $h \in \operatorname{Ker} f$.

Remark. For any normed linear space $X$, each nonzero vector $x$ has a norming hyperplane.

Proof. Let $x$ be any nonzero vector. Let $r=\|x\|$. Let $U$ be the open ball of radius $r$ centered at the origin. Let $C$ be a closed convex set disjoint from $U$ such that $\{x\}=C \cap \bar{U}$. By the Hahn-Banach theorem, there is a continuous linear functional $f$ such that $|\operatorname{Re} f(u)|<\|x\|$ for all $u \in U$, and $|\operatorname{Re} f(y)| \geq\|x\|$ for all $y \in C$. Clearly, $\|f(x) \mid=\| x \|$ and $\|\operatorname{Re} f\|=1$. It can easily be verified that $x+\operatorname{Ker} f$ is a norming hyperplane at $x$.

Theorem 11. Let $(X,\|\cdot\|)$ be a strictly convex smooth reflexive Banach space. Let $\|\cdot\|_{1}$ and $\|\cdot\|_{2}$ be two equivalent norms on $X$ such that $\|\cdot\|_{1} \leq\|\cdot\|$ and $\|\cdot\|_{2} \leq\|\cdot\|$. Clearly, for each $x_{0} \neq 0$ and for each $0<\epsilon<\left\|x_{0}\right\|$, the closed ball ${\overline{B\left(x_{0}, \epsilon\right)}}_{\|\cdot\|}$ is weakly compact in $\left(X,\|\cdot\|_{i}\right)$ for $i=1,2$. So, $\|\cdot\|_{1}$ and $\|\cdot\|_{2}$ attain their minimum over ${\overline{B\left(x_{0}, \epsilon\right)}}_{\|\cdot\|}$ at some points of ${\overline{B\left(x_{0}, \epsilon\right)}}_{\|\cdot\|}$. Suppose, for each $x_{0} \neq 0$ and for each $0<\epsilon<\left\|x_{0}\right\|,\|\cdot\|_{1}$ and $\|\cdot\|_{2}$ attain their minimum over $\overline{B\left(x_{0}, \epsilon\right)} \|_{\|\|}$at the same point. Then, $\|\cdot\|_{1}=K \cdot\|\cdot\|_{2}$ for some constant $K$.

Proof. Let $S=\left\{x \in X\right.$ : for some $f \in X^{*}$, the hyperplane $x+\operatorname{Ker} f$ is norming at $x$ w.r.t. both $\|\cdot\|_{1}$, and $\left.\|\cdot\|_{2}\right\}$. We will show that $S$ is dense. For any nonzero vector $x_{0}$ and any $0<\epsilon<\left\|x_{0}\right\|$ let $v_{x_{0}, \epsilon}$ be the point in $\overline{B\left(x_{0}, \epsilon\right)}$ at which both $\|\cdot\|_{1}$ and $\|\cdot\|_{2}$ attain their minimum over $\overline{B\left(x_{0}, \epsilon\right)}$. The set of all such $v_{x_{0}, \epsilon}$ is dense. We will show that $v_{x_{0}, \epsilon} \in S$ for each $x_{0}$ and $\epsilon$ with $0<\epsilon<\left\|x_{0}\right\|$. Let $f \in X^{*}$ be such that $\|\operatorname{Re} f\|=\left\|v_{x_{0}, \epsilon}\right\|_{1},|\operatorname{Re} f(x)|<\left\|v_{x_{0}, \epsilon}\right\|_{1}$ for all $x \in B\left(v_{x_{0}, \epsilon},\left\|v_{x_{0}, \epsilon}\right\|_{1}\right)$ and $|\operatorname{Re} f(x)| \geq\left\|v_{x_{0}, \epsilon}\right\|_{1}$ for all $x \in \overline{B\left(x_{0}, \epsilon\right)}$. Then, $v_{x_{0}, \epsilon}+\operatorname{Ker} f$ is a norming hyperplane at $v_{x_{0}, \epsilon}$ w.r.t $\|\cdot\|_{1}$. If this hyperplane is not norming at $v_{x_{0}, \epsilon}$ with respect to $\|\cdot\|_{2}$, then $\left\|v_{x_{0}, \epsilon}+h\right\|_{2}<\left\|v_{x_{0}, \epsilon}\right\|_{2}$ for some $h \in \operatorname{Ker} f$. So, for some 
$t>1, \quad\left\|t\left(v_{x_{0}, \epsilon}+h\right)\right\|_{2}=\left\|v_{x_{0}, \epsilon}\right\|_{2}$. The line segment joining $t\left(v_{x_{0}, \epsilon}+h\right)$ and $v_{x_{0}, \epsilon}$ is contained in the closed ball of radius $\left\|v_{x_{0}, \epsilon}\right\|_{2}$ centered at the origin. Let us observe that for some $\lambda>0$ small enough, the vector $\lambda \cdot\left(t v_{x_{0}}+h\right)+(1-\lambda) \cdot v_{x_{0}} \in$

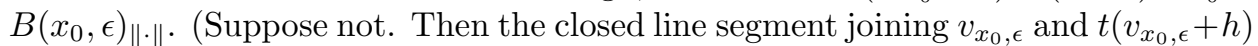
is disjoint from the $\|\cdot\|$-open set $B\left(x_{0}, \epsilon\right)_{\|\cdot\|}$. So, there is a $\|\cdot\|$-closed hyperplane containing this line segment and disjoint from $B\left(x_{0}, \epsilon\right)$. Clearly, this hyperplane is different from $v_{x_{0}, \epsilon}+\operatorname{Ker} f$. But there is a unique supporting hyperplane at $v_{x_{0}, \epsilon}$. This contradiction implies the assertion.) That is, $B\left(0,\left\|v_{x_{0}, \epsilon}\right\|\right)_{\|\cdot\|_{2}}$ intersects with $B\left(x_{0}, \epsilon\right)_{\|\cdot\|}$. This contradicts the hypothesis that $\|\cdot\|_{2}$ attains its minimum over $\overline{B\left(x_{0}, \epsilon\right)}$ at $v_{x_{0}, \epsilon}$.

Next, let $x$ be any nonzero vector in $X$, and let $\delta>0$ be arbitrary. Let $\epsilon>0$ be such that $\frac{\delta}{1+\delta}>\frac{\epsilon}{1-\epsilon}$. It is easy to check that if some $y \in B(x, \epsilon)$ admits a norming hyperplane, then that norming hyperplane is $(1+\delta)$-norming at $x$. Thus, each nonzero vector in $X$ admits a $(1+\delta)$-norming hyperplane for each $\delta>0$.

Note that if $x+H$ is $(1+\delta)$-norming at $x$, then for any closed subspace $N$ with $H \cap N \neq\{0\}, x+(H \cap N)$ is also $(1+\delta)$-norming at $x$ in the span of $\{x, H \cap N\}$.

If the conclusion of the theorem is not true, then there exist $x$ and $y$ in $X$ such that $\|x\|_{1}=K \cdot\|x\|_{2}$ and $\|y\|_{1}=K(1+\epsilon) \cdot\|y\|_{2}$ Consider the two-dimensional space $N$ spanned by $\{x, y\}$.

If the conclusion of the theorem is not true, then there exist $r_{i}>0$ for $i=1,2$ such that $B\left(0, r_{1}\right)_{\|\cdot\|_{1}} \cap N \not \subset B\left(0, r_{2}\right)_{\|\cdot\|_{2}} \cap N$ and $B\left(0, r_{2}\right)_{\|\cdot \cdot\|_{2}} \cap N \not \subset B\left(0, r_{1}\right)_{\|\cdot\|_{1}} \cap N$. It can be seen that there exists some $x \in \partial\left(B\left(0, r_{1}\right) \cap N\right) \cap \partial\left(B\left(0, r_{2}\right) \cap N\right)$ which does not admit a hyperplane in the two-dimensional space, which is $(1+\delta)$-norming w.r.t. both norms $\|\cdot\|_{1}$ and $\|\cdot\|_{2}$.

\section{REFERENCES}

1. B. Beauzamy, Introduction to Operator Theory and Invariant Subspaces, North-Holland (1988). MR 90d:47001

2. M. I. Kadets, On spaces isomorphic to locally uniformly rotund spaces, Izv. Vysš. Učebn. Zaved. Mathematika 1959, no. 6, 51-57. (Russian) MR 23:A3987

3. S. Mazur, On the generalized limit of bounded sequences, Colloquium Mathematicum, 2, (1949-1951), 173-175. MR 14:159k

4. H. Radjavi and P. Rosenthal, Invariant Subspaces, New York: Springer-Verlag, 1973. MR 51:3924

Department of Mathematics, Kent State University, Kent, Ohio 44242

Current address, Shamim Ansari: Department of Mathematics \& Statistics, Drawer MA, Mississippi State University, Mississippi State, Mississippi 39762 